\title{
Belphégor
}

\section{S/Z (Les impressions du Zigomar de Léon Sazie)}

\section{Paul Bleton}

\section{(2) OpenEdition}

\section{Journals}

\section{Édition électronique}

URL : http://journals.openedition.org/belphegor/157

DOI : 10.4000/belphegor.157

ISSN : 1499-7185

Éditeur

LPCM

\section{Référence électronique}

Paul Bleton, « S/Z (Les impressions du Zigomar de Léon Sazie) », Belphégor [En ligne], 11-1 | 2013, mis en ligne le 28 avril 2013, consulté le 21 décembre 2020. URL : http://journals.openedition.org/ belphegor/157 ; DOI : https://doi.org/10.4000/belphegor.157

Ce document a été généré automatiquement le 21 décembre 2020.

\section{(c)}

Belphégor est mis à disposition selon les termes de la Licence Creative Commons Attribution - Pas d'Utilisation Commerciale - Pas de Modification 4.0 International. 


\title{
S/Z (Les impressions du Zigomar de Léon Sazie)
}

\author{
Paul Bleton
}

\section{NOTE DE L'AUTEUR}

La recherche dont ce texte est issu a été rendue possible par une subvention du Conseil de recherches en sciences humaines du Canada. Elle a abouti dans un essai, $L a$ Cristallisation de l'ombre (2011) et plusieurs articles. Merci aux passionnés du forum « À propos de littérature populaire » http://litteraturepopulaire.winnerbb.net/ pour les nombreuses informations glanées dans leurs échanges ainsi qu'à Bérengère Vachonfrance-Levet et Désiré Nyela pour leurs commentaires.

\section{Le Z d'amnésie}

[...] Z est la lettre de la mutilation : phonétiquement, $\mathrm{Z}$ est cinglant à la façon d'un fouet châtieur, d'un insecte érinnyque; graphiquement, jeté par la main, en écharpe, à travers la blancheur égale de la page, parmi les rondeurs de l'alphabet, comme un tranchant oblique et illégal, il coupe, il barre, il zèbre [...] (p. 103-104) ${ }^{1}$

1 Roland Barthes (1970) nous avait clairement et éloquemment prévenus contre les maléfices de cette lettre. L'avertissement devrait d'ailleurs valoir a fortiori pour Sazie mieux adapté à cela qu'un Sarrasine ou qu'un Balzac puisque l'orthographe même de son nom conjoint au $\mathrm{Z}$ inverseur la lettre inversée, sa majuscule initiale... Contrairement à l'effeuillage d'un texte classique lexie par lexie faisant apparaître le rôle structurant de quelque castration cachée, face à Zigomar et Sazie, c'est plutôt, sous la cruelle lumière médiatique, l'oubli dans lequel ils ont été relégués qui aveugle. Oh, pas complet, sans doute: de tardives entrées pour l'auteur et sa créature dans le Dictionnaire des littérature policières (2003), le Dictionnaire du roman policier 1841-2005 (2005), le Dictionnaire du roman populaire francophone (2007), les deux dernières évoquant le substantiel article de Dominique Kalifa paru en 2005, «Zigomar, grand roman sériel 
(1909-1913) », plusieurs mentions de l'importance négligée de Zigomar au service de l'Allemagne dans l'histoire de la fiction d'espionnage française dont celle dans La Cristallisation de l'ombre (2011) du signataire de ces lignes... Le tout sent néanmoins l'embaumement faute d'avoir convaincu un éditeur de la pertinence d'une réédition, un siècle plus tard.

\section{Faux-départ}

2 Pourtant, si l'on en juge par sa fortune médiatique, transmédiatique même, les choses n'avaient pas si mal commencé pour le scélérat à la cagoule. Victorin Jasset, au sommet de sa brève mais prolifique carrière largement consacrée à l'adaptation filmique de fictions populaires, l'avait même adopté. Avec ses précédentes "Aventures de Nick Carter » (1908), Jasset avait non seulement tiré pour les films Éclair, qui venait de l'engager, la matière de la célèbre série (inaugurée en 1886 par John R. Coryell, portée par sa propre collection fasciculaire, Nick Carter Weekly, et diffusée en traduction française par Eichler), mais il s'en était aussi inspiré pour inventer la sérialisation de la fiction filmique - d'abord une histoire complète chaque fois mais avec le retour des mêmes personnages principaux, puis le «feuilleton» cinématographique. Par l'abaissement de l'importance de l'auteur, de son autorité (elle paraissait anonymement), cette série américaine encourageait, emblématisait même un tel mode de production : l'histoire de départ aurait été suggérée à Coryell par Ormond G. Smith, fils d'un des propriétaires de Street \& Smith, l'éditeur de dime novels, elle a été poursuivie avec de nombreuses mutations par de nombreux auteurs dont la signature n'apparaissait pas plus sur les fascicules (outre Coryell, Frederick Van Resselaer Dey, Thomas C. Harbaugh, Eugene T. Sawyer, Richard E. Wormser) et adaptée plusieurs fois à l'écran et pour les ondes dans les années 40 aux État-Unis...

3 C'est avec une désinvolture comparable à l'égard de l'œuvre originale que Jasset croise l'univers criminel de Sazie - Zigomar, roi des voleurs (1911)2, Zigomar contre Nick Carter (1912) et Zigomar, peau d'anguille (1913), ce dernier en trois époques, avec Alexandre Arquillère en Zigomar ${ }^{3}$, André Liabel en Paulin Broquet ${ }^{4}$ et Josette Andriot en Rosaria ${ }^{5}$. Désinvolture qui conduit Sazie, géographiquement plus proche de l'infidèle que les auteurs d'un Nick Carter parisianisé, à désavouer cet avatar de son Zigomar, à dénoncer l'abus, à poursuivre Éclair... Compréhensible, cette mauvaise humeur ne rend pourtant pas justice à l'effet de sidération que devait provoquer dans les salles obscures la danse du feu-follet de Mlle Esmée au Moulin rouge - danse à la Loïe Fuller, colorisée et accompagnée de musique - l'irruption de la technologie au service de la violence l'avion en est à ses débuts à l'écran et Zigomar, peau d'anguille intègre à la trame narrative des évolutions aériennes (et explosives) du fameux pilote Attilio Maffeï - ou la brutale cruauté de l'invention - Paulin Broquet a beau fermement camper du côté de la loi, de l'ordre et de l'axiologie des spectateurs, ayant appris le suicide par empoisonnement de son vieil adversaire finalement capturé par Nick Carter, il n'en demande pas moins un abject mémento à un médecin de l'hôpital hébergeant provisoirement le "cadavre»: un morceau de peau de Zigomar pour s'en faire un portefeuille (dans La Résurrection de Zigomar, premier des trois épisodes de Zigomar, peau d'anguille, 1913) : frissons assurés !...

4 Sans en être la cause, cette mauvaise humeur a sans doute aussi contribué à faire des Zigomar de Jasset un faux départ. Il pourrait être suggestif de rapprocher leurs dates de 
celle des Mystères de New York. On sait que, à partir de l'œuvre originale publiée dans Cosmopolitan par le journaliste Arthur B. Reeve (une série de 82 nouvelles policières inspirées de celles de Sherlock Holmes) ${ }^{6}$, à l'initiative de Pathé Exchange, Louis Gasnier, George B. Seitz et Leopold Wharton avaient tourné un serial de 36 épisodes, réduit à 22 pour la consommation française - la formule allait permettre à Charles Pathé de maintenir la production alors que toute l'industrie cinématographique ressentait en France les effets de la mobilisation générale. On sait aussi que ces 22 épisodes devaient avoir un corrélat imprimé, Pierre Decourcelle en publiant la novellisation dans le Matin, en 22 épisodes parallèles, du 27 novembre 1915 au 28 avril 1916. Faux départ des Zigomar de Jasset parce qu'ils ne sont pas portés par un modèle d'affaire intégrateur comme celui de Pathé, emprunté à William N. Selig - il avait expérimenté la stéréomédiation film-journal du serial avec le Chicago Tribune de William Mac Cormick en 1913. Aux États-Unis, Pathé avait d'abord signé une entente avec William Randolph Hearst, ce qui lui ouvrait de la publicité dans une centaine de journaux de villes américaines importantes ${ }^{7}$; avec le Matin, il allait se contenter d'implanter en France une formule qui avait fait ses preuves. Suite au succès du premier serial, Les Exploits d'Elaine allaient en constituer une suite, accompagnée comme son ombre par le cinéroman de Marc Mario. Alors que la sérialisation mise au principe de la création, romanesque puis filmique, aurait pu s'instituer en modèle dès les Zigomar de Jasset, ni le réalisateur ni Éclair n'avaient entrevu cette communauté d'intérêt entre le cinéma et le journal alors que c'est elle qui inventera un objet médiatique nouveau, hybride, perpétué à l'initiative des studios et des journaux: le journal utilisé pour maximiser impact et profits d'un film.

$5 \quad$ Faux départ des Zigomar de Jasset aussi parce que la mort prématurée de Jasset en 1913, suite à une intervention chirurgicale, et les déboires de la troisième firme de production française (incendie de ses studios américains à Fort Lee, décision de Maurice Tourneur et Émile Cohl de rester en Amérique après la guerre, problèmes financiers) n'auraient pas pu consolider l'avance de Jasset, encore moins perpétuer une formule « prédatrice » sans-gêne, uniquement fondée sur l'adaptation, face à la formule de la novellisation et du ciné-roman.

6 Faux départ des Zigomar de Jasset enfin, mais à un titre moindre, parce que, malgré leur succès puis celui des films de Louis Feuillade, des Fantômas à Tih Minh, Les Mystères de New York auront implanté un modèle de narratologie filmique pour le cinéma criminel primitif dérivé partiellement $\mathrm{du}$ mélodrame via le roman-feuilleton. Le terme melodrama pour Hollywood renverra moins à Pixerécourt qu'il ne désignera un genre pathétique (ou sentimental) fondé sur les péripéties "catastrophiques ou providentielles" advenant à une Victime et se déclinant en crime melodrama, war melodrama, etc. ${ }^{8}$ Dans le modèle melodrama, non seulement le spectateur perçoit-il les événements surtout à travers la compréhension qu'en a l'héroïne, non seulement celleci est-elle la victime, quelle que soit la nouvelle situation de chaque nouvelle bande, non seulement a-t-elle l'assistance indéfectible mais pas toujours très efficace de son fiancé, mais elle est en butte aussi à un Méchant tapi dans l'ombre dont le spectateur a toutefois une bonne idée. Alors que, du roman de Sazie, c'est thématiquement plutôt le manichéisme et l'hydre toujours renaissante du scélérat que Jasset tirait. Or la précoce interruption du proto-serial allait lui dénier la consistance qui s'acquiert dans l'attente du titre suivant; Protéa sérialisée mais pas adaptée d'un roman préalable allait remplacer un Zigomar à succès mais décidément trop récalcitrant; en détective patriote et adepte de jiu-jitsu, Josette Andriot, en collant et en Paul Poiret, du côté du 
Bien, allait aisément supplanter Alexandre Arquillère en cagoule, du côté du Mal, dans les salles obscures... et dans les rêveries érotiques des soldats revenus du front en permission?.

7 Une fois Jasset croisé, la fin des Zigomar devait aussi sceller la carrière de Sazie au cinéma; il ne semble rester de lui qu'une continuité dialoguée non réalisée, Humanité (s.d.), proposée à (ou commandée par) Albatros, la compagnie de production francorusse mise sur pieds par Alexandre Kamenka en 1920 dans les studios de Montreuil rachetés à Pathé.

Versons deux dernières pièces au dossier de la célébrité du pourtant furtif et insaisissable criminel. Dans son impressionnante collection de plaques autochromes couleur, les «Archives de la Planète » du Musée Albert-Kahn à Boulogne-Billancourt disposent d'un cliché pris le mardi 14 mai 1918 par Auguste Léon, l'un des photographes employés par le banquier mécène, montrant la modeste façade du Grand Cinéma Plaisir et sa petite marquise, 95 rue de la Roquette dans le $11^{\mathrm{e}}$, où Zigomar est à l'affiche. Sous le regard de badauds, ce sont même plusieurs affiches, deux en format portrait et en noir et blanc et deux en format paysage et en couleur, qui débordent, occupent la vitrine aux rideaux de fer clos du commerce voisin, les Chaussures Bully ${ }^{10}$. Quant à Philippe Melot (1997), il évoque d'indubitables indices de notoriété, emblèmes de la métabolisation gourmande de Zigomar par la société française : des effigies en pain d'épices et en sucre candi vendues dans les fêtes foraines.

\section{Ambivalence ? Méconnaissance?}

Et pourtant, Zigomar est bien oublié. On ne saurait certes reprocher ni à lui ni à son $\mathrm{Z}$ leurs prédécesseurs ou leur postérité ; mais comment ne pas se souvenir du Zigomar de Léon Gandillot? Un commissaire, un gendarme et un magistrat ont beau paraître sur la scène du Théâtre du Palais Royal, le Zigomar princeps n'est pas propre à effrayer : le surnom ne fait qu'affubler de l'indice de fêtard un Amédée provincial qui jette sa gourme à Paris avant le mariage. Pauvre premier Zigomar précipité dans l'imbroglio d'une pantalonnade vaudevillesque ; pauvre premier Zigomar (1900) qui ne tint l'affiche qu'une malheureuse semaine ${ }^{11} . .$. Et comment ne pas noter le coefficient de ridicule affectant la mégalomanie du Zorglub signant d'un $\mathrm{Z}$ qui, depuis 1961 et le quinzième volume des aventures de Spirou et Fantasio d'André Franquin et Greg, oblige à lire à partir de la droite tous les mots des dialogues en zorglangue, elle-même enseignée en accéléré (selon la suggestopédie de Georgi Lozanov) ? Comment ignorer un plus récent avatar, pas aussi effrayant que Sazie aurait pu le souhaiter, apparu dans le livre pour enfants de Philippe Corentin, Zigomar n'aime pas du tout les légumes et il a bien raison (1992) ?

10 Il faut dire que le nom commun lui-même manque décidément de dignité, fût-ce pour désigner un sanguinaire scélérat. Attesté avant ce dernier, zigomar dériverait de zig ("homme, zigoto", individu épateur: «faire son zigoto", dès 1902) agrémenté du suffixe argotique - mar usuel dès 1800 (guichmar = guichetier ; boss'mar $=$ bossu ; offic'mar = officier) selon Gaston Esnault (1919). Pire encore : le lexicographe ne se contentant pas de la facile paternité du terrible Zigomar de Sazie révise l'étymologie, non sans quelque insolence pour le criminel, Zigomar le zigouillard:

Le nom de ce meurtrier, quoiqu'il ne fût pas sabreur, a-t-il suffi pour faire baptiser

le sabre de cavalerie? On peut supposer un syllogisme plus précis. Majeure: 
allusion à la marque $\mathrm{Z}$ que porte un certain sabre, comique par le suranné de son modèle, ce sabre des pompiers [...] que portaient aussi, au début de la guerre [1914-1918], les infirmiers et brancardiers, le sabre nommé par la théorie sabre série $Z$. Mineure : allusion à ce fait, copieusement réitéré dans le roman de M. Sazie, que l'assassin en chef Zigomar et la bande des Zigomars ses séides ont pour signe de reconnaissance la lettre majuscule $\mathrm{Z}$, soit découpée, soit brodée sur des cagoules, ou collée aux murailles, ou dessinée d'un geste dans l'air, ou tracée du doigt sur une table. Conclusion SABRE (série $\mathrm{Z}$, ou autre) $=$ Zigomar. [...] Si on ajoute à ce sémantisme que le lieu, peut-être unique, où le mot a été observé est un dépôt de l'ouest, il est vraisemblable que les cavaliers avaient déjà nommé leur sabre un zigue ou un zigouillard; ce n'est plus seulement une lettre initiale, mais un vrai radical, qui se trouvait alors tout prêt à recevoir la terminaison -omar. (p. 547-548)

11 Alors, Zigomar? Tragique ou rigolo ? Zig-zag d'une ambivalence ? Est-ce dans ce nom hésitant entre deux registres qu'il faudrait repérer le germe de son oubli ? La durée, le succès et la fortune transmédiatique du feuilleton de Sazie, les témoignages de l'époque concordent pourtant: les lecteurs ne trouvaient pas Zigomar ridicule. Peut-on par ailleurs rappeler que l'impertinence parodique n'a pas été épargnée à Fantômas, notamment celle du feuilleton radiophonique Signé Furax (1956-1960) de Pierre Dac et Francis Blanche, largement décliné ${ }^{12}$, bien mieux réussi que les films d'André Hunebelle $^{13}$, et que ce ridicule ne l'a pas tué pour autant. Peut-on aussi rappeler que Zigomar n'est pas le seul à avoir subi ce cruel destin? Que l'on se souvienne du plus étique succès et du plus triste sort de son émule Ténébras, le bandit fantôme d'Arnould Galopin, porté par un format plus ample $(19 \times 27 \mathrm{~cm})$ mais aussi plus modeste (42 livraisons de fascicules de 16 pages), fascicules illustrés par E. Bouard ${ }^{14}$.

Serait-ce donc chez Léon Sazie (1862-1939) lui-même, né en Algérie et mort accidentellement à Suresne, qu'il faudrait chercher un fêle originel ? Philippe Melot parle à son propos d'une « vieille famille basque béarnaise » (p. 85). Pourtant, les traités de phonologie tiennent pour caractéristique du basque le fait qu'un mot ne peut contenir à la fois une consonne sifflante apicale (l'articulation se fait avec la pointe de la langue) et une consonne sifflante laminale (elle se fait avec les bords de la face supérieure de la langue) : sasi [un roncier] est possible, pas sazi*... Créateur du scélérat masqué, voilà Sazie le Basque débasqué, en quelque sorte...

13 Pas assez sérieux? Dans son brûlot antisémite Les Juifs en Algérie (1888) ${ }^{15}$, Georges Meynié rapportait, avec beaucoup de pugnacité mais aussi de flou, la tragique histoire d'un M. Sazie, notable oranais ruiné dans la faillite frauduleuse de son banquier, M. Lévy ; le notable se fit sauter la cervelle dans le bois du Planteur. Vraisemblablement, il s'agissait respectivement du président et du trésorier de la Chambre de commerce de la ville. Or la mère de Léon Sazie semble avoir été Mme Eugénie Laisant, veuve de VictorAntoine Sazie, le suicidé, mère de six enfants et sœur de l'ex-capitaine Charles-Ange Laisant, attaché à la direction du génie à Tlemcen en 1875. Selon le journal satirique bimensuel lyonnais la Comédie politique (28 mai 1882), Eugénie Laisant vendait en 1882 pour 90000 francs-or ${ }^{16}$ les vastes mines de Maaziz (1110 ha, produisant à cette époque de la calamine, du zinc, du plomb argentifère) près de Tlemcen, obtenues par décret du 23 juillet $1875^{17}$ en dépit des habituels refus de l'administration aux demandes de concessions: possible passe-droit accordé au capitaine du génie, qui en aurait fait profiter son beau-frère. Pour tempérer la verve du satiriste, «habituels refus de l'administration " semble une expression un tantinet exagérée si l'on en juge à ce que révèle le Bulletin officiel du Gouvernement général de l'Algérie, qui en répertorie une dizaine pour cette même année 1875 . Plausible, le soupçon s'assortit pourtant mal à la suite de 
la carrière de Charles-Ange Laisant, cinq fois élu député républicain après sa démission de l'armée, de 1876 à 1893, à Nantes puis Paris, boulangiste en 1889, puis anarchiste après sa carrière politique, un temps directeur du Petit journal de Moïse Millaud, le plus gros tirage des journaux parisiens (à partir de 1879), docteur en mathématique dont le nom est attaché à un nombre hypercomplexe, le quaternion ${ }^{18}$.

Au-delà de ça, je dois avouer mon ignorance sur la vie de Sazie. Le possible suicide du père pourrait déterminer la mort comme fausse sortie pour Zigomar, parfaitement répétable? Il existe bien un portrait de Sazie par Louis-Edouard Brindeau de Jarny, peintre orientaliste à la fois son contemporain et son " pays " maghrébin ${ }^{19}$; mais je ne connais pas ce portrait du créateur d'un scélérat sans visage ou aux visages multiples. Savoir que Sazie s'y entendait en épée ne mène pas très loin non plus. Il ne s'y entendait pas simplement à cause de tel commentaire éclairé et autorisé sur tel duel, à propos duquel on pourrait toujours rétorquer qu'il est fictif (celui entre le comte de la Guérinière et Marc Collas, le premier ayant demandé réparation au second, journaliste, pour un entrefilet laissant entendre sa culpabilité dans l'affaire Montreil, dans Zigomar); mais aussi parce que Sazie tirait lui-même à l'épée et qu'il devait même inventer la pointe d'arrêt à trois branches, assurance arbitrale que le coup avait porté quoiqu'inoffensive pour les escrimeurs. Cela n'aura guère aidé sa cause après la marginalisation du duel, migré de la vie sociale et du statut de passion française vers le statut bien plus périphérique de sport ${ }^{20}$. L'invention romanesque laisse entendre que Sazie avait accompagné cette migration ; entre Paulin Broquet et Zigomar, on se trouve à mi-chemin entre compétition et point d'honneur devant laver les offenses dans le sang. Enfin, à défaut d'en connaître la teneur, que faire des nombreuses interventions de Sazie au Club du Faubourg scrupuleusement minutées par Léo Poldès, son fondateur?

Rien dans tout ce fatras ne peut encore éclairer ni a fortiori expliquer une telle débauche de méchanceté de son criminel, une telle amnésie quant à Sazie.

\section{Hors de Zigomar, point de salut}

16 Avant Jasset, Sazie ne semble avoir qu'assez peu profité de la convergence des fictions populaires imprimées et filmées, si ce n'est que, lors de la sortie du court-métrage qu'en avait tiré Henri Andréani pour la Société des Grands Films Populaires de Georges Lordier en 1913, Le Petit Journal avait réédité Jacques l'honneur, un drame co-signé avec Georges Grison ${ }^{21}$, joué au théâtre République Château-d'Eau en 1894 puis gonflé en « roman dramatique » de plus de 1300 pages en 1895.

17 Après Jasset, Sazie n'est plus que parcimonieusement adapté, surtout par comparaison avec les romanciers populaires contemporains qui avaient investi la transmédiatisation et avec la fécondité de la compétition entre Pathé et Gaumont. Fantômas, Rocambole, Les Mystères de New York, Les Vampires, Les Exploits d'Elaine, Judex, Tih Minh... : la rivalité incite en fait à explorer quelques unes des possibilités narratives du film criminel en train de naître et des formes d'adaptation et de novellisation. Sazie reste en dehors de cette arène. Enfants de Paris, son roman le plus adapté et novellisé est un mélodrame - un film muet d'Albert-Francis Bertoni en 1924, avec Félicien Tramel et Marguerite Madys, dont Sazie signe la novellisation pour la collection Cinéma bibliothèque de Tallandier, une réédition du roman dans Le Livre national de Tallandier en 1930, un remake parlant de Gaston Roudès en 1936, avec Paul Bernard et Lisette Lanvin. 
Il raconte l'histoire des amours partagées et contrariées de Ginette, la simple ouvrière, et d'André, apparemment simple ouvrier mais en réalité fils de patron. Le père de l'une ne transige pas sur l'honneur de sa fille ; le père de l'autre le destine à la perpétuation de l'entreprise et au mariage avec la fille de son associé. L'une croit à l'infidélité de l'autre, s'enfuit, tombe malade ; la fiancée putative mais compréhensive lève le malentendu, rabiboche les amoureux et les pères nobles cessent de les désespérer.

Et c'est un conte, «La légende de la Cueva del agua » - initialement paru à Oran en 1889, narrant les amours de Pépé, un jeune jardinier poète et musicien, et de Pépita, tous deux captifs du bey d'Oran : en fuite, ils se réfugient dans une caverne attendant l'embarcation des contrebandiers qui doit leur permettre de rentrer en Espagne, risquent d'y périr de soif jusqu'à ce qu'une source d'eau douce les sauve - qui sera adaptée en pièce radiophonique sous le titre «La source d'amour ", par le poste de la Tour Eiffel (2 février 1931).

Avant Zigomar, Sazie avait tâté de la scène. Outre Jacques l'honneur, il y avait eu Bisbis de ménage (1893), une niaise comédie.

Dans un élégant salon, une querelle de deux jeunes mariés, Max et Berthe : pour elle, persécutrices fumées de la lampe, de la cheminée, du cigare, à quoi elle répond par le thé ; pendant l'absence de l'autre, chacun goûte l' « arme » de l'adversaire, il boit du thé, elle tire sur le cigare, la réconciliation est totale !

Il y avait eu Un gendre au cassoulet (1893) - un certain Boucabeille, de Narbonne, remarque à propos des dames de la capitale : «Et c'est avec ça... en sachant seulement bavarder, broder, chanter, pianoter... et pas de cassoulet... qu'elles croient faire le bonheur d'un mariage, vos femmes de Paris! ». Il y avait eu La Sonate en mi (1893), «Je suis discrète ", un monologue recueilli dans Le Livre des petites filles (1893), Larmes d'or et gâteau de plomb (1898) de la même eau, pour jeunes filles, et, co-signé avec Grison, le vaudeville Caponet, Pipard et Cie (1896).

Deux associés dans une affaire de chocolaterie se prétendant d'une grande respectabilité alors que l'un flirte avec une actrice, l'autre avec une employée : premiers rendez-vous galants, pris à l'insu de l'autre partenaire mais au même endroit, justement déserté par Mme Caponnet partie en voyage, accident de train inattendu, retour de Mme Caponnet et des domestiques, vif jeu de cache-cache pour planquer les maîtresses...

Avant Zigomar, Sazie avait aussi tâté du texte court dans les journaux et les magazines, le Pierrot, les suppléments du Petit Journal et de la Vie illustrée; après le succès de son scélérat, il publiera des « romans-concours » dans Je sais tout...

À l'époque de Zigomar, c'est avec un autre détective superlatif, Martin Numa, flanqué de son Watson, le journaliste Courville, que Sazie approche à la fois le récit criminel et la sérialisation, dans un journal de faits divers illustrés qui marquera la période 1908-1914, L'Æil de la police ${ }^{22}$. Après Zigomar, Mirobal puis Bochemar déçoivent; initialement parus dans le Journal, ni l'un ni l'autre ne seront repris en volume. Le premier (1913-1914) surnomme ainsi le détective Tony Pacot qui lutte contre Jim 
Schader et la bande des X; le second (1916) reprend le suffixe à succès en contexte patriotico-guerrier, pour une histoire d'espionnage où le capitaine de zouave Robert Darney s'oppose à Bochemar, un espion allemand aidé de la Gitane Marfa et du baron von Krapman. Malgré une campagne publicitaire pour le lancer ${ }^{23}$, ces données en font trop un émule du Naz-en-l'air de Souvestre et Allain, datant de 1912-13, pour s'imposer.

Après la Grande Guerre, dans une période pourtant riche pour le roman d'aventures, Sazie n'y trempera que le bout de la plume : il y avait petitement commencé en 1903 et à partir de 1925 allait donner de petits romans aux collections de Ferenczi, Les Romans d'aventures, Le livre de l'aventure, Voyages et aventures, sous son nom ou sous divers pseudonymes comme Captain Hidden, Joë Traveller... Du côté du roman policier, hormis deux petits romans signés Noël de Saize parus dans «Les Maîtres du roman populaire " de Fayard au début des années $30^{24}$, il se contentera de rééditions; c'est Tallandier qui allait reprendre en volumes les Merveilleux exploits de Martin Numa, le roi des policiers en 1931, dans la collection Espions, criminels et policiers, où les 10 titres de Sazie étaient encadrés par des séries de Gabriel Bernard - ils succédaient aux 19 premiers titres des Aventures de l'inspecteur Tony et précédaient Satanas et Les nouvelles aventures de l'inspecteur Tony. C'est surtout au roman sentimental et pathétique et à un lectorat féminin que Sazie devait consacrer l'entre-deux-guerres. Il y avait là aussi commencé tôt avec Chérie et maudite (1897) et Un Double amour (1914). Il alimentera les collections de Ferenczi (Les Romans choisis, Les Grands romans), Tallandier (Le Livre de poche, Le Livre national, Romans célèbres de drame et d'amour), Fayard (Les Maîtres du roman populaire, Le Livre populaire), avec des titres ne risquant guère de lui apporter l'immortalité, brillant plutôt par leur typicité générique: Mensonges de femme, La Voleuse de bonheur, Le Martyre de Lucienne, La Martyre blonde laquelle appelait une suite idoine, L'Aurore du bonheur, Mon coeur est à toi, La Conquête d'une âme, L'Amour fait souffrir, L'Amour de la gamine, J'ai payé son cœur, La Gitane amoureuse, La Femme trompée " roman dramatique », suivi des Larmes de la vie...

Sazie n'y croyait-il plus vraiment? Romancier d'un seul livre, jamais ré-égalé, dont l'imaginaire avait passé de mode, supplanté et relégué aux oubliettes par son émule Fantômas ? Ou, dans un registre plus cynique, échaudé par la désinvolture du cinéma, dans le confort du roman sentimental et pathétique, aurait-il négligé d'assumer le service après-vente qu'un Marcel Allain, plus fécond et un peu plus diversifié, offrira à Fantômas? Trop de zones d'ombre empêchent encore de répondre ; aussi autant partir de l'élusif et sinistre criminel central, des textes qui en construisent la menaçante histoire ; autant les prendre par le biais de l'acte de lecture, de son double dynamisme centripète et centrifuge et par celui de sondages suggérés par un mot lui aussi protéiforme ${ }^{25}$.

\section{Première impression}

D'un côté, il y a le mouvement: la culture médiatique, une force qui entraîne, l'obsolescence rapide programmée placée au principe de fictions substituables les unes aux autres, de feuilletons dont la suite arrive fidèlement au prochain numéro. Avant même Zigomar, en voici le nom distillé comme une liqueur d'énigme. En novembre et décembre 1909, par l'indéchiffrable répétition de plus en plus fréquente de ce seul nom, Zigomar, chaque fois imprimé en caractères différents, puis par l'annonce à la fois explicite et prodigue d'épithètes «Très prochainement, Le Matin publiera Zigomar par 
Léon Sazie. Ce roman, où les pires bandits et les policiers les plus illustres luttent sans trêve, à travers la capitale, est une sorte d'Épopée du Crime. Des figures de femmes, mères douloureuses, fiancées tragiques, ouvrières parisiennes, créature de grâce et d'amour, dominent ce drame haletant. Zigomar, c'est le roman de toutes les aventures, où sont dévoilés les effroyables dessous des drames les plus palpitants. », puis par le dessin de l'inquiétant encagoulé au $Z$, enfin, le décembre par le rappel «Demain mardi Le Matin publiera Zigomar par Léon Sazie » encadré avec le dessin, composite, de la pensivité détective devant le cadavre recouvert d'un $\mathrm{Z}$ (évoquée plus haut) et d'une vignette de jeune femme en buste, le quotidien Le Matin crée l'attente chez ses lecteurs $^{26}$. En fait, il faudra prendre la place laissée libre en bas de la page 2 par la fin du feuilleton de Gaston Chérau, Le Remous. Ce qui s'effectuera le 7 décembre, avec dès les premières trois colonnes une fracassante entrée en matière en trois temps : un incipit généralisant « Dans Paris retentit ce matin un cri d'indignation générale, de terreur, de colère. ", son immédiate restriction à la médiation de la presse qui rapporte l'assassinat sanglant du banquier Monteil, poignardé, et un double kérygme - celui des deux adversaires dont la lutte structurera le récit, Paulin Broquet, « le plus habile, le plus fin des inspecteurs de la Sûreté, le plus célèbre des détectives français » qui en démontre la justesse en récupérant une mystérieuse signature sanglante, peu visible sur la porte du coffre vidée, un $\mathrm{Z}$ aussitôt commenté par le clairvoyant Broquet dans un registre communicationnel, « Exprès pour nous, ce Z... pour nous, la justice... pour les autres, les complices... peut-être aussi pour la victime !... », commentaire creusant dans l'esprit du lecteur la niche vide pour un Zigomar déjà prévisible. 164 feuilletons plus tard, cette première histoire terminera, déplacée en bas de la page 4 du journal, le dimanche 22 mai 1910, sur la victoire très incomplète de Broquet, le corps inerte de Zigomar emporté sous ses yeux jusqu'à la puissante voiture de course de Thom Tweak par "cette merveilleusement belle, cette étrange inconnue, cette mystérieuse femme rousse » sur laquelle il ne peut se résoudre à tirer puisqu'elle lui avait sauvé la vie chez Zulma - fin du « livre troisième, L'Heure de la justice ».

On est loin de la littérature majuscule de l'époque, du roman à thèse de Paul Bourget, de la création de la Nouvelle Revue française ou de la parution de La Porte étroite d'André Gide, pourtant strictement contemporains ; dynamique, certes, ce Zigomar fait bien mauvaise impression. Outre ce mauvais goût, la suite de la vie éditoriale de ce divertissement d'ilotes assume sa nature de marchandise. Après le roman-feuilleton, l'histoire du terrible Zigomar paraît hebdomadairement en 1909-1910 chez Ferenczy (lequel a encore son y pour ce Z), en 28 fascicules de 128 pages illustrés par Georges Vallée, subdivisée en une prolifération de sous-titres - Zigomar: Le maitre invisible, Zigomar: Les lions et les tigres,. Zigomar: L'heure de la justice, La Femme rousse: Le bras marqué, La Femme rousse: Dans l'au-delà, La Femme rousse: My Darling, Peau d'anguille: L'homme inattendu, Peau d'anguille: Au plus fort! Elle sort ensuite en deux forts volumes, en 1916, chez un Ferenczi (auquel le z cinglant, peut-être, a fait perdre son y), sous des couvertures de Gino Starace et les titres Zigomar et Zigomar au service de l'Allemagne devenu autonome, ce dernier rallonge Peau d'anguille de quelques chapitres de mise en place. En 1922 et 1923, le même Ferenczi redécoupe la matière narrative (et l'allège subrepticement) dans sa nouvelle collection Les Romans choisis, avec quelques autres titres de Sazie ${ }^{27}$, sous des couvertures de Henri Armengol, Zigomar, Zigomar: Riri la jolie, Zigomar: La femme rousse et Zigomar: My darling. Intérêt relancé du lectorat pour la cruelle crapule? Volonté de l'éditeur de presser le citron jusqu'à la dernière goutte ? Nostalgie de Sazie? Toujours est-il qu'un nouveau roman, Zigomar contre Zigomar paraît 
en 1924, en huit livraisons, sous une couverture à la plastique repensée par Armengol : un $\mathrm{Z}$ rouge et carré barre entièrement la couverture y délimitant deux zones distinctes hébergeant deux scènes séparées.

\section{Couverture d'un des facicules de Zigomar contre Zigomar}

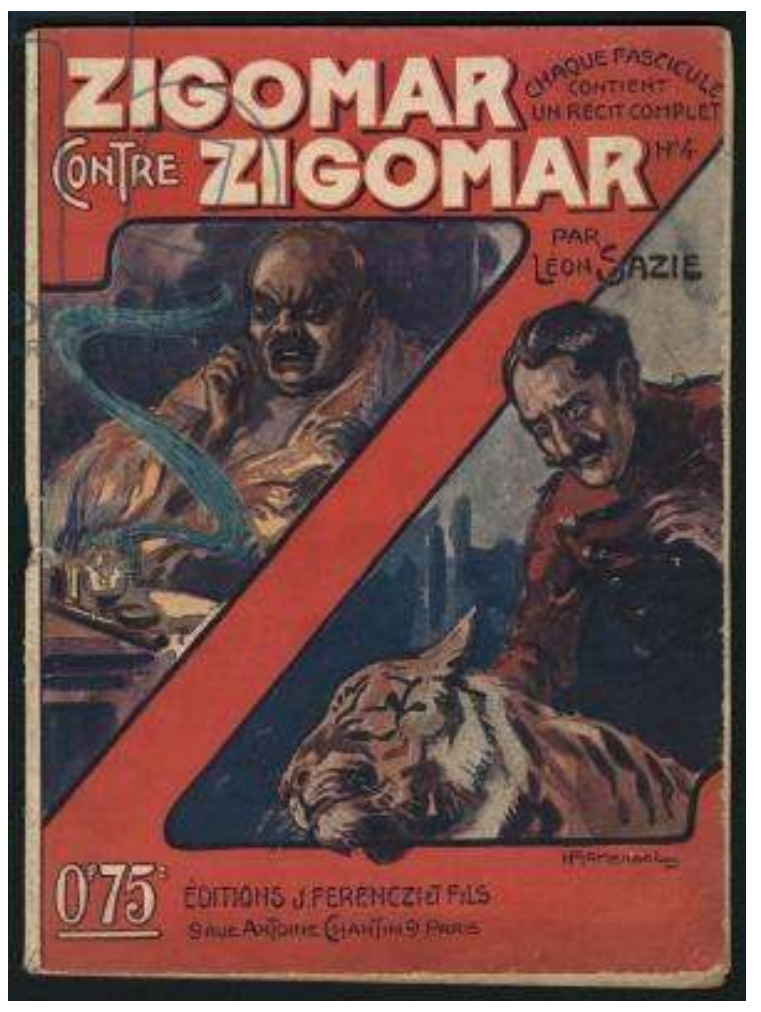

Ferenczi, 1924

Quant au dernier avatar, initialement paru en feuilletons dans le Petit Parisien en 1938, Un Nouveau coup de Zigomar, c'est Tallandier qui l'offre en volume, posthumément, en 1948, dans la collection rouge du Livre national. Protéiforme : la valse-hésitation des identités n'affecte pas seulement le sombre héros masqué mais tout aussi bien le récit de ses calamiteux exploits.

Amateur de Zigomar, Apollinaire ne l'en trouvait pas moins «écrit n'importe comment ». Sans doute pas seulement à cause du mode de parution en feuilletons mais aussi parce que les intrigues se multiplient et que l'auteur saute un peu arbitrairement de l'une à l'autre en donnant, souvent mais pas toujours, des explications apostérioriques. La tolérance du lectorat devant l'apparente incohérence des enchaînements devait être grande, exercée par ces résumés des épisodes précédents proposés par les fascicules, résumés certes moins lus que les feuilletons et les chapitres. Philippe Mellot (1997) en cite un (en fait tiré de Peau d'anguille: Au plus fort!) qui vaut effectivement son pesant de zigomars en sucre candi :

Paulin Broquet sachant que l'homme de la Morgue est non Bénamol, mais Schmidt, cherche à trouver la filière qui relie la bonne allemande grâce aux œufs en celluloïd aux bandits. Puis ayant entendu Fernande faire avant de mourir un terrible aveu, il sait quels ennemis il a à combattre. Simonet porte les documents secrets en Angleterre, mais Paulin Broquet veille, fait retrouver le plan. Zigomar est blessé par lui sur le toit d'un wagon. Les trois amis passent en Angleterre, où les $\mathrm{Z}$ essayent en 
vain d'arracher sous la torture de la presse à lithographie, au capitaine de la Rochardie, le secret des documents... conscience sous ce verre grossissant d'une caractéristique de l'esthétique de Sazie ? Ainsi, en l'espace de quelques feuilletons, le lecteur est introduit à une foule de personnages et à quelques uns des nombreux fils de l'écheveau narratif. Raymonde, la fille du banquier mystérieusement assassiné, fiancée au capitaine Fabien de Rennebois, sœur de Robert le médecin (le docteur Robert pour les pauvres qu'il soigne gratis) et de Raoul l'avocat, se voit en outre pourvue de deux amies, Irène et Alice de Brialle, laquelle a perdu sa mère, son frère et sa petite sœur d'une maladie mystérieuse et foudroyante; c'est Robert qui avait pratiqué l'autopsie de la petite. Voici maintenant Riri-la-Jolie, jeune et exquise ouvrière accompagnant le couturier chargé de façonner les vêtements de deuil ; elle est la sœur de Marie la bossue et fille d'une pauvresse que le docteur Robert vient d'accepter de soigner. Sans encore connaître leurs sentiments respectifs, les deux frères sont amoureux de Riri-la-Jolie. Pour le lecteur à qui on ne la fait pas, sans qu'il soit nécessaire de le dire explicitement, Marcelin, valet bien suspect, espionne la famille Montreil dans le deuil. Les funérailles de M. Montreil sont peu suivies, au grand dam de ses deux fils... L'acte de lecture est interrompu par l'alternance des différents fils narratifs, à la manière de Ponson du Terrail. Il faut préciser que, quoique datant du Second Empire ${ }^{29}$, le cycle de Rocambole était en cours de réédition dans la collection Le Livre populaire d'Arthème Fayard, entre 1908 et 1912. À la frustration du lecteur laissé ainsi en suspens au moment où tel fil est abandonné (provisoirement, espère-t-il) s'ajoute une légère désorientation au moment de se raccorder à tel nouveau fil. Sans compter que tous ces fils ne sont ni également développés, ni leurs syntagmes disjoints développés à un rythme égal, ce qui suscite au moins la perplexité du lecteur, ouvre ce que Barthes nommait des codes. À son fiancé, le capitaine de Rennebois, Raymonde a annoncé qu'elle ne l'épouserait qu'après avoir résolu l'assassinat de son père : symbolique encadrement du désir. Décès rapides dans la famille d'Alice: possible émergence d'une énigme? Deux frères parallèlement amoureux de Riri-la-Jolie: possible risque d'activer le topos des frères ennemis? Premiers soupçons quant au valet Marcelin avant l'expédition nocturne des deux frères chez le notaire; nouveaux soupçons à leur retour qui expliqueraient comment c'est leur poignard qui a frappé le gardien de nuit : pour le compte de qui ? à quelles fins? Les funérailles boudées, provisoirement inexplicables mais vécues comme un camouflet à l'honneur de la famille Montreil : pourquoi ?

Nous lisons un tel texte aujourd'hui à travers le filtre du genre policier, qui s'est initialement constitué par un resserrement du récit autour de l'enquête, formule dominante même si celle de Maurice Leblanc (une énigme actuelle trouve sa solution en se superposant presque terme à terme sur une histoire criminelle ancienne) a exercé un attrait pendant plus d'un siècle. Un tel resserrement renvoie aux vieilles lunes du roman baroque la perpétuelle fuite en avant narrative, la relative indifférence à la cohérence, alors même que l'invention romanesque populaire, de Ponson à Sazie, y puisait son inspiration. Sans y parvenir entièrement, elle cherchait à détourner les yeux du lecteur de la tentation d'une lecture mimétique, à harnacher le maximum de strates sémiotiques qui auraient pu inciter à la dispersion de son attention au profit d'un noyau signifié simple, axiologique, à abaisser la description au profit d'une prédilection pour le dialogue; en quoi elle se distinguait du roman réaliste. En y parvenant plus complètement, elle instaurait l'activisme maniaque du récit, 
l'inépuisable perpétuation du plaisir, le « et après ? et après ?... » mobilisant l'attention du lecteur, la maintenant tendue ${ }^{30}$.

31 Pour cette école de l'invention romanesque, le récit ne saurait avoir de fin. La disparition du corps de Zigomar serait le propre de l'interminable selon Sazie, son Z non pas une fin mais un nouveau départ - le moment boustrophédon du retournement Zigomar/Ramogiz. Le récit pointe explicitement cette insolite strate de sens, alphabétique, en plaçant le $\mathrm{Z}$ en vedette. Signature énigmatique, on l'a vu. Signature dont la perception se mérite : parmi les représentants des forces de l'ordre réunis sur la scène du premier crime, seul Broquet détecte le premier $\mathrm{Z}$ sanglant chez le banquier assassiné, puis le second perceptible par une mince pellicule de sable sur le coffre-fort violé du notaire cambriolé, sur la scène du second crime. $Z$ dont le texte qualifie pour le lecteur la fonction de signature et la valeur perlocutoire :

« Ce Z de sang et ce $\mathrm{Z}$ de sable qui se trouvaient là comme signature de l'auteur des drames, ainsi que le disait justement Paulin Broquet, prenaient un caractère merveilleux, angoissant, terrible... » (19 déc. 1909) ${ }^{31}$.

32 Le $\mathrm{Z}$ sert aussi de signe de ralliement. Lors de sa première occurrence, le lecteur constate qu'un signe à la fonction simple, comme l'emblème, peut non seulement avoir une archéologie (chez Clafous, rencontre du patron avec deux ouvriers de la construction, ils trinquent, le texte explique : « Ces deux mots : Zalamor... Zalavi... que les amis venaient d'échanger mystérieusement, étaient une des plaisanteries classiques de l'ancien mélo des théâtres du boulevard du Crime... Quand le héros du drame s'écriait: "Je suis à toi jusques à la vie!» les autres répondaient: "Moi z’à la mort !... » ", 6 janv. 1910) mais aussi, selon l'universalité de la maxime des apparences trompeuses dans cet univers, un double fond - il s'avérera en effet que si chez Clafous il $\mathrm{y}$ a bien réunion des $\mathrm{Z}$, pour le lecteur les deux premiers à proférer le mot de ralliement sont en fait des policiers déguisés !

33 Enfin, le $\mathrm{Z}$ indique où doit se placer la majuscule, dans quel sens doit se faire la lecture $\mathrm{du}$ mot ramogiz. Espionné par Broquet dans la carrière souterraine qui sert de lieu de réunion aux $\mathrm{Z}$, Zigomar apparaît au lecteur pour la première fois, en majesté et son discours s'entend dûment ponctué à l'exclamation :

Nous sommes les Ramogiz! Ramogiz que par tradition, renversant le nom, nous appelons Zigomar ... ! Zigomar ! c'est le cri des Ramogiz ! C'est le cri qui a traversé les siècles! C'est le nom de celui que personne n'a vu, ne connait mais dont tout le monde sent la puissance... C'est le nom de celui qui, immortel comme le soleil, peut se renouveler chaque jour, être différent, tout en restant le même, mourir sans arrêter de vivre, s'éteindre sans cesser de briller, c'est Zigomar !... (9 janv. 1910)

Déclaration épidictique saluée par le $\mathrm{Z}$ des Ramogiz agité en guise de rameaux, en quelque sorte. Placé devant au moins quatre registres interprétatifs possibles, que choisit le lecteur $?$ Le registre mimétique explicite et centrifuge, Ramogiz = Gitans, c'est-à-dire l'appel à la vieille hantise européenne, datant de l'arrivée des Tziganes au $\mathrm{XV}^{\mathrm{e}}$ siècle, du voleur d'enfants, nomade malveillant partout infiltré fondant le modèle de toute contre-société illégale, au principe de l'invention de l'argot ${ }^{32}$ ?... Le registre symbolique de ce Montmartre souterrain, avec son labyrinthe de protection, la mortelle salle du Minotaure, ancienne carrière de gypse oubliée, l'égout passant par un des couloirs pour aller jusqu'à la Seine et servant à dépêcher les condamnés du tribunal inquisitorial des $\mathrm{Z}$, les juges encagoulés, l'interprétant religieux d'un lieu qui en impose, ("religieux silence», «le moindre bruit prenait sous ces voûtes une ampleur énorme et résonnait comme dans une cathédrale. », 8 janv. 1910) ? Un registre plus 
flou, empruntant aux précédents et à bien d'autres sources, comme la nouvelle rapportée ces jours-là par le Matin de l'ouverture de la ligne de métro Porte de Clignancourt-Porte d'Orléans passant sous la Seine : circulation souterraine légitime, saut technologique vers la modernité, en face d'une autre, inquiétante, illégale, malfaisante, mais occupant le même sous-sol urbain ? Ou le registre centripète à quoi pousse l'activisme maniaque du récit: Broquet subrepticement mais imprudemment encagoulé parmi les $\mathrm{Z}$ encagoulés se voit désigné par Zigomar, dénoncé, vilipendé par les Ramogiz, reconnu comme un adversaire coriace par Zigomar lui-même, puis lâchement précipité dans le puits souterrain conduisant à l'égout encore plus souterrain? Pas le temps de tellement s'arrêter aux Tziganes et à leur place dans la société française, on ne garde qu'une vague impression d'eux, vite décalée sur leurs synonymes substituts, Ramogiz et $\mathrm{Z}$. Une scène chasse l'autre. Filant sur le toboggan diégétique le lecteur s'inquiète du sort de Broquet, bientôt redoublé par celui du brave mais imprudent Clafous déguisé en sergent de ville, lui aussi bientôt reconnu, emmuré par une explosion, blessé de surcroît par le lâche coup de poignard d'un Z...

Chargé de la première impression, le journal donne moins une impression de confusion - qui pourra être éventuellement attribuée aux résumés de la secrétaire peu scrupuleuse d'un éditeur reprenant en fascicules la pré-publication en feuilletons qu'une impression de mouvement perpétuel, de vitalité vivace, d'allant énergique.

\section{Mauvaise impression}

D'un autre côté, il y a la lutte contre l'impermanence : la fiction en culture médiatique tente de résister à l'obsolescence de plus en plus rapide des récits. Parlant d'impression, un contemporain comme Henri Avenel (1901) souligne l'importance matérielle de l'amélioration et de la disponibilité des encres, notamment celles de la maison Charles Lorilleux, et des innovations d'Hyppolite Marinoni en fait d'impression, presse rotative à retiration sur papier continu, à partir de $1872^{33}$. Dès lors, l'impression bascule dans l'ambivalence: de fixation encrée, la voici passée par la rapidité des rotatives à produire du texte journellement obsolescent. Négligeant les auteurs, pour qui l'industrie parasitant leurs romans-feuilletons afin de leur donner une seconde vie était à la fois une source de revenus et une machine d'aliénation, tournons-nous de nouveau vers ce que percevait l'acte de lecture.

Tout d'abord, dans la matérialité du texte elle-même, puisque la réédition de romansfeuilletons en fascicules, en volumes, est une tentative de freiner cette obsolescence, à défaut de pouvoir s'y opposer. Outre l'ampleur textuelle, c'est l'instauration de la couverture et la promotion de l'image qui affichent le mieux le changement de vecteur. Même en papier-journal, la couverture offre au roman-feuilleton une sorte d'élévation, rapprochant le fascicule du livre, de l'imprimé de prestige. Certes, les images avaient accompagné Zigomar dès ses origines feuilletonesques. Dans la campagne de lancement, le Matin avait déjà eu recours au signe vide (le dessin d'un inquiétant encagoulé émergeant d'un $\mathrm{Z}$ sanglant en forme de faux) et aux cercles concentriques de ce pavé dans la mare (une jeune femme, l'innocente persécutée de l'increvable tradition mélodramatique ?... un penseur perplexe devant le cadavre recouvert d'un $\mathrm{Z}$, le criminel allant se manifester aussi bien par d'homicides effets que par sa seule apparence). 
A fortiori, la lutte contre l'impermanence recourra à la couverture en couleurs lors de la publication en volume, les 28 fascicules de 128 pages $11 \times 14 \mathrm{~cm}$. Comme le reste de l'impression, l'illustration de couverture est marquée par l'ambivalence. Dessins et couleurs de Georges Vallée annoncent au chaland à quoi s'en tenir ; immédiatement perçue, accrocheuse, elle drague le client qui passe. Sensationnelle, elle saisit souvent une action à son acmé : l'image est à la fois immédiatement lisible et fréquemment sibylline. Elle invite ainsi à lire ce qui va se passer. Inscrit en son principe se trouve déjà le moment où la page de couverture tournée, ne restent plus devant le regard que les pages d'écriture; mais le temps où elle est sous le regard, quel punch! Au moment du tir, une déflagration se produit sur le servant d'une puissante pièce d'artillerie: maladresse, accident, sabotage? Dans le désordre d'un accident de la circulation en ville qui a attiré des badauds, un homme en uniforme (de chauffeur ?) assène de la tête un puissant coup de bélier à un autre homme en uniforme: qui sont-ils pour se retrouver ainsi spectaculairement en conflit? Un homme couché attaché sur un banc, bâillonné, poitrine nue, un énorme explosif à la mèche allumée fixé sur le corps, à ses pieds un $\mathrm{Z}$ rouge : explosera-t-il ? et, subsidiairement, que s'est-il passé pour qu'il se retrouve dans la bizarrerie de cette funeste situation?

Zigomar, première série

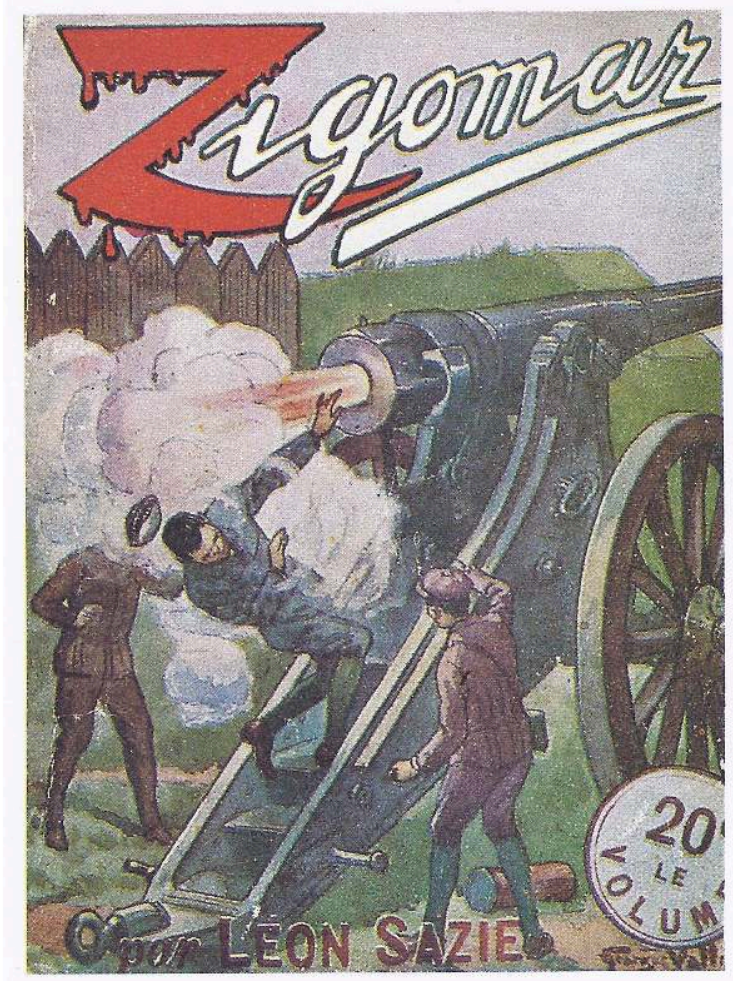

Ferenczy, 1909-1910

Parfois, désobligeante roublardise de lecteur $\mathrm{du} \mathrm{XXI}^{\mathrm{e}}$ siècle ?, l'énigme cède la vedette à la perplexité. Une poursuite en voiture dont les occupants se tirent dessus; la calandre de la De Dion-Bouton (de la Renault, de la Delaugère \& Clayette, de la Darracq, de la Zèbre... ?) poursuivie fonce vers le lecteur, ce qui renvoie autant au contenu à venir du roman qu'à une imagerie rendue commune par le journal de criminalité motorisée, une nouvelle forme de la peur associée à la modernité. Laquelle est un tantinet anticipée 
par Sazie et Vallée: en 1903, il y avait bien eu la course-poursuite menant à l'arrestation de la bande à Jacob, l'anar illégaliste dont l'esprit lors de son procès avait séduit le journaliste Maurice Leblanc ${ }^{34}$, mais il faudra attendre le 21 décembre 1911 et l'attaque de la succursale de la Société Générale de la rue Ordener à Paris par la bande à Bonnot pour l'invention du hold-up à moteur... Ou encore, cette illustration, dont on appréciera les courts-circuits, d'une opposition anthropologique fondamentale, le nu et le cuit : un homme nu mais à la moustache conquérante, sorti du bain, tente d'en noyer un autre, vêtu, dans l'eau très chaude de la baignoire: en fait, l'homme nu est un personnage en uniforme, le capitaine La Rochardie, qui prend le dessus sur un soldat sans uniforme (un espion allemand qui tentait de lui subtiliser un document secret à l'hôtel) cruellement ébouillanté tout habillé dans la baignoire - qu'on se rassure, le malheureux réussira malgré tout à s'enfuir...

Zigomar, première série

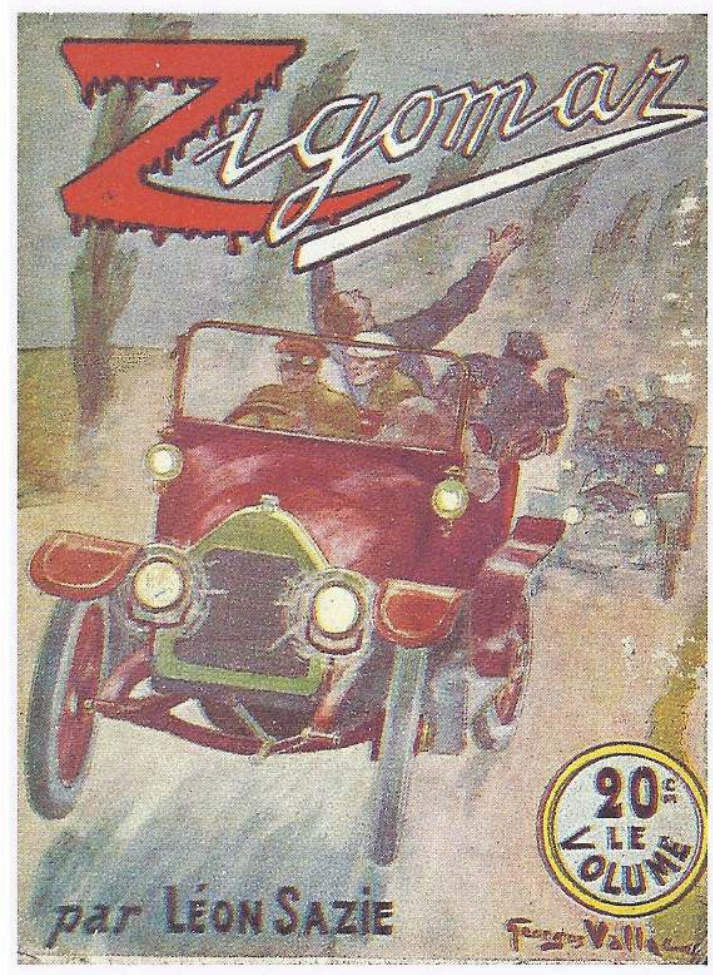

Ferenczy, 1909-1910

L'acmé se déplace parfois de l'action à ses effets saisissants et tragiques. Au théâtre ou à l'opéra, des voisins découvrent horrifiés un spectateur en costume de soirée poignardé, l'arme encore dans son plastron et sa poitrine. Les portes ouvertes d'une penderie révèlent l'alignement de deux hommes encadrant une femme, tous élégamment vêtus, tous pendus par le cou aux crochets de la penderie. Sur une table Louis XVI, le cadavre encore fumant, d'un homme vêtu couché dont la cagoule et le visage ont disparu (sous la morsure de l'acide ?) exhibe hideusement son crâne décharné, alors que gît sous la table un autre homme inconscient (mort?) un marteau à la main et que, à côté d'eux, un coffre-fort repose sur une couverture (tentative de le forcer ?) 


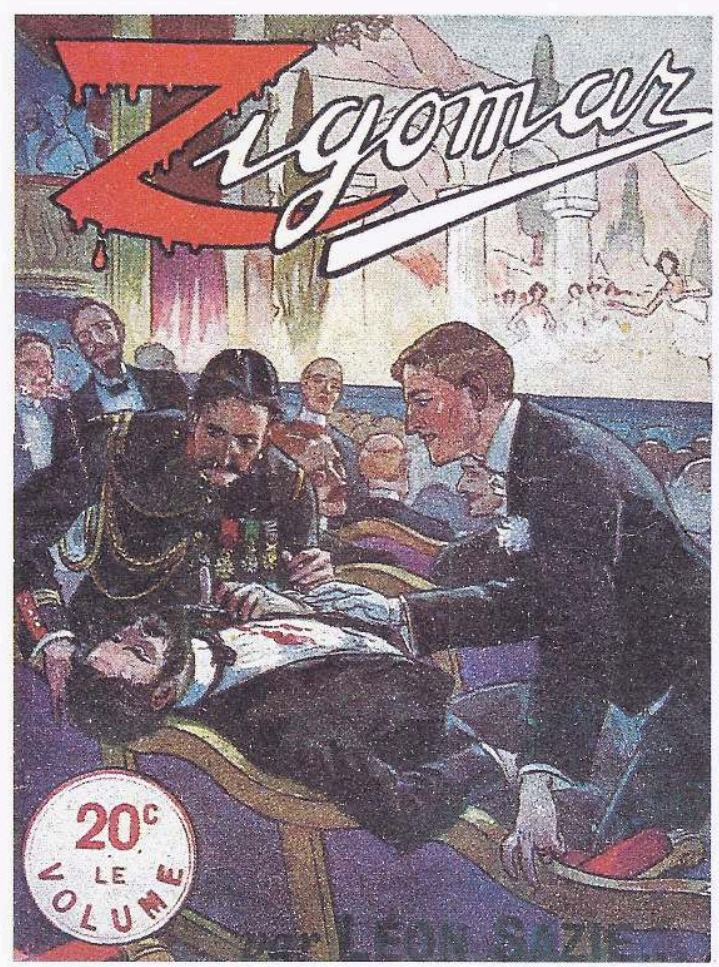

Ferenczy, 1909-1910

41 L'illustration ne sert pas seulement à accrocher le lecteur; il lui arrive aussi de le leurrer. Deux hommes en uniforme de fonctionnaire sont fauchés par un cycliste en uniforme de groom qui a perdu le contrôle de sa bicyclette : plus simple que l'accident précédemment mentionné, puisqu'elle ne semble impliquer qu'un seul incident alors que l'autre impliquait accident préalable suivi de bagarre. Or, à la lecture, le lecteur verra toutefois que les apparences peuvent être trompeuses puisque cet accident, provoqué, sert le projet malveillant du pseudo-groom, en réalité la comtesse Warsovia... 


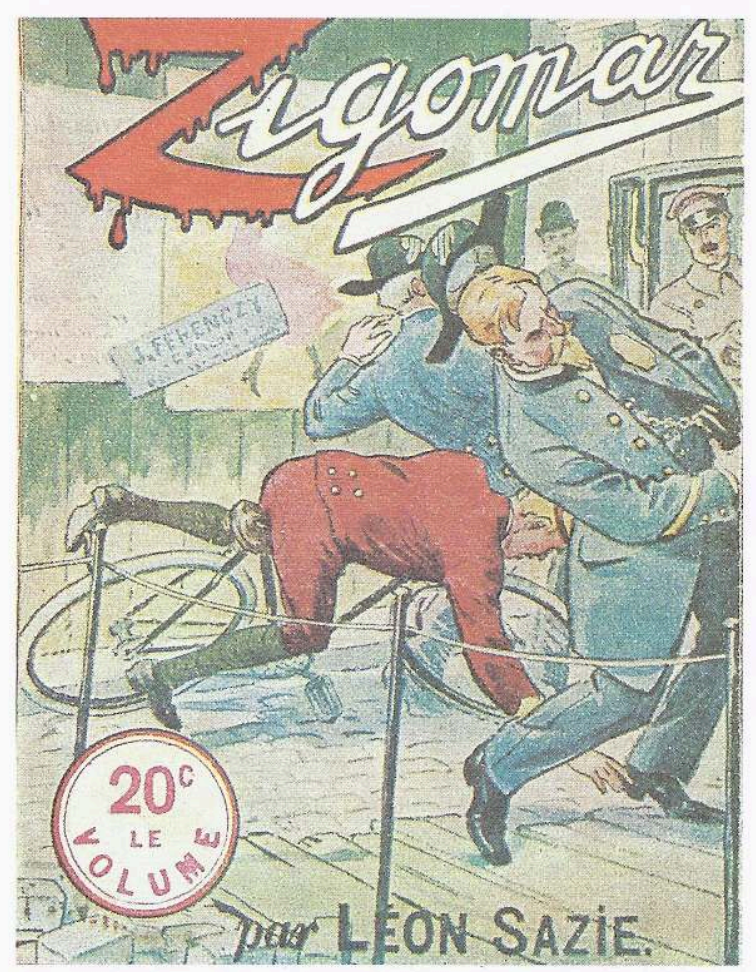

Ferenczy, 1909-1910

Dans ce registre, les images les plus placides s'avèrent vite les plus retorses. L'intérieur tranquille d'une brasserie (on distingue uniquement des choppes sur la table et sur le plateau du garçon) où l'on discute et fume paisiblement - la lecture amènera son lecteur à insister par réversion sur les barbes, la rousseur, la pipe et le costume du personnage en avant-plan : sur sa coupable germanité. Volatiles et autres animaux de compagnie en cage, la vendeuse du magasin et sa cliente visiblement sous le charme d'un ara perché sur son juchoir : l'intérieur d'une oisellerie, quoi de plus serein? La lecture ajoutera ce qui ne peut se voir et en renverse complètement les apparences, un système d'écoute clandestin mettant en péril la sécurité du pays. 
Zigomar, première série
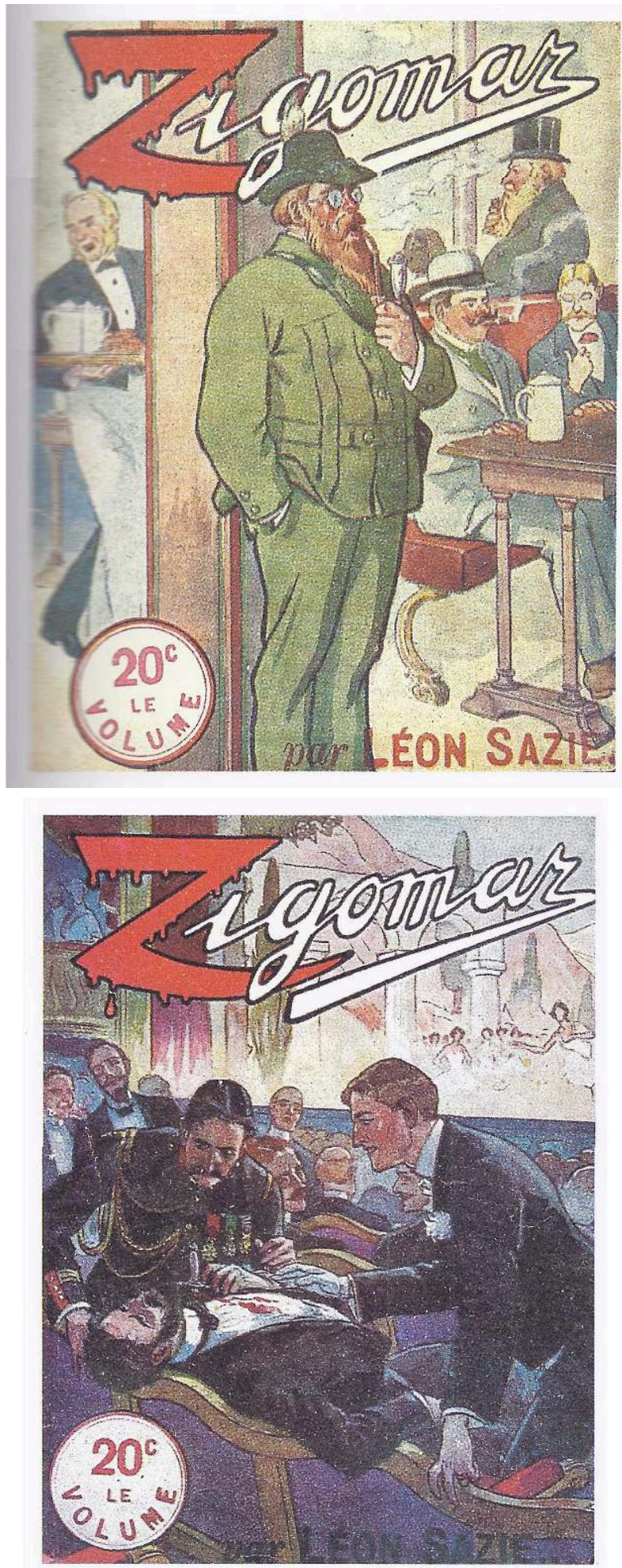

Ferenczy, 1909-1910 sournoisement imposer une inquiétude. Celles mettant en scène Zigomar entraînent le lecteur comme un invisible courant: la cagoule fantomatique, vers le registre gothique; la cagoule pénitentielle, vers le registre religieux; le costume en cuir 
couvrant entièrement le personnage avec une cape-cagoule, de grosses lunettes, vers le registre de la modernité, c'est son exosquelette que ce zig emprunte au homard...

Outre la station sur l'icône marquant le seuil, le récit se charge de représenter la lenteur pour le lecteur, de personnifier les aléas de la coopération interprétative. Paulin Broquet est une machine sémantique triphasée. Première phase, " homme de goût, artiste et sportsman accompli ", haltérophile, escrimeur, boxeur, maître en jiujitsu, cavalier émérite, sachant conduire toutes sortes de machines, bon pilote et yachtman, polyglotte, "très au courant des mouvements des lettres... des théâtres ", amateur de musique, pianiste honorable et peintre à ses heures: Broquet cumule des compétences. Deuxième phase, il lui faut un court-circuit de la pensée pour reconnaître après-coup dans l'élégant maître de cérémonie du duel, le "si décoratif baron Dupont ", l'ouvrier alcoolique qui s'en était pris au comte de la Guérinière que Broquet conduisait chez M. Montreil pour la confrontation initiale ! À quoi l'un des assistants de Broquet ajoute que ce même transformiste incarnait aussi un ami du comte. Troisième phase, synthèse d'acquisition de compétences et de perception au-delà des apparences, voici Broquet en déchiffreur qui décode des instructions données par Zigomar à l'un de ses sbires et un lieu de rendez-vous, mais sans expliquer comment; puis qui élucide un autre message, plus sibyllin, sur le verso de la feuille « MENE... BARONE...COLIE... SOIR.. BAL... MAHON. » (1 janv. 1910), ce qui lui permet de donner à ses aides et au lecteur une leçon de lecture comme choix d'interprétations plausibles. Il y a sans doute chez lui le rythme de l'action, le réflexe décisif, l'immédiate perception; mais la lenteur est le rythme nécessaire à jauger les personnages, à interpréter les situations et à éventuellement errer, à démêler les écheveaux, à réagir aux mauvais coups de l'adversaire scélérat, à débrouiller les secrets, à percer à jour les mystères. Un exemple d'errements, tiré du premier livre, Le Maître invisible, qui commence par une dénégation: avant la confrontation du banquier grièvement blessé et de l'homme qu'il accuse, Broquet tend sa cravache à ce dernier, le comte Faustin de la Guérinière ${ }^{35}$, suspect du meurtre de $\mathrm{M}$. Montreil, de telle sorte que le comte l'empoigne par la main gauche... et l'échappe. Inférence de Broquet le rusé, qui tire de l'incident visiblement plus que ce que son lecteur aurait pu faire : cette maladresse met le comte hors de cause puisque les coups de poignard meurtriers avaient été portés de la main gauche. Pourtant le comte n'en est pas moins accusé : confrontation à la fois confondante et tragique puisque à l'accusation formelle est suivie d'une inexplicable rétractation, transformée en énigme par le décès du banquier... Or, si la ruse de Broquet disculpait le comte, pourquoi diable ce revirement non pas directement du détective mais du comte: "Arrivé près de la porte, il se retourna et lança au détective un coup d'œil railleur de triomphe et de défi. Paulin Broquet répondit à cette bravade par un regard ferme, mais froid et tranchant, comme sont les lueurs des épées mises au clair » (10 déc. 1909) ? Un exemple d'énigme dont la résolution devra longuement attendre, tiré du même livre: Octavie Bilmant jeune a épousé un placier en produits chimiques, Albert Laurent, malheureusement spéculateur, et faussaire - il a engagé toute la dot de son épouse, et la fortune de son avare de beau-père qui ne lui a jamais pardonné d'avoir épousé sa fille. Combat entre les $\mathrm{Z}$ dirigés par leur chef et Broquet qui les surprend en train de voler le beau-père d'Albert, beau-père qui vient de refuser son aide financière à ce gendre pris à la gorge par une échéance ; les malfrats réussissent à s'enfuir. Concomitamment, le comte de la Guérinière qui a demandé réparation au journaliste se bat en duel avec lui ! Comment le comte, soupçonné d'être Zigomar, peut-il en même temps diriger une bande de truands en Normandie et se battre devant témoins à Paris? 

version améliorée. Devant la débauche des identités et des apparences, avouez que pour tout autre que lui, super-lecteur partiellement manipulateur de la réalité truquée, on peut y perdre son latin. Ou plutôt, tomber sur des grumeaux de sens qui font parfois un peu trébucher la lecture, laissant au lecteur l'impression d'avoir maîtrisé une difficulté. Dans un couloir secret chez Clafous, "Antonin redevenait ici Paulin Broquet, après avoir laissé en bas le fidèle Gabriel, qui dans le rôle de Gustave, filait le baron Dupont en père de famille aveyronnais... L'homme qui l'attendait devant la petite porte était l'Amorce... Clafous était réellement Clafous, mais il faisait partie de la compagnie, de la brigade de Broquet. » (7 janv. 1910). La prestesse de Zigomar ressort d'autant mieux sur l'alternance de vivacité de l'action et de fonctionnement parfois laborieux de la machine sémantique triphasée que le lecteur ne peut que supputer l'avoir sous les yeux. Zigomar n'a pas à démonter ses tours de prestidigitation. Telle serait d'ailleurs une caractéristique déterminante du Méchant ici : contrairement à Monte Cristo ou Rio Santo dans l'intimité des intentions de qui le lecteur ne se trouve qu'occasionnellement mais qui sont facilement compréhensibles par leur statut de vengeur, contrairement à un converti comme Rocambole, chez Sazie le héros s'interpose entre lecteur et Méchant, il introduit une distance narratologique, ses inattentions, ses incompréhensions, ses erreurs de jugement, sans parler des réticences et de la mauvaise foi du narrateur, perturbent la juste compréhension du lecteur ou la retarde ${ }^{36}$.

Du côté de l'acte de lecture, sans que l'on puisse parler d'ambivalence comme dans le cas des illustrations de couverture, il faut noter que, quels que soient les efforts du texte pour précipiter le lecteur, pour lui faire dévaler le toboggan diégétique, bien des tentations se présentent en cours de lecture, qui en freinent le rythme, choix alternatif d'autres registres d'interprétation, vagabondages induits par un texte qui n'arrive pas à harnacher la rêverie de son lecteur rétif ou dissipé...

Parisien et contemporain de Sazie, ou, plus tardif et informé par Le Montmartre du plaisir et du crime (1980) de Louis Chevalier, le lecteur ne peut que rapprocher, par une digression mimétique, Clafous (ou du moins le personnage qu'il joue) d'un historique et brutal cabaretier-indicateur.

Rêveur et saisi par la description de la crypte-cathédrale souterraine des $\mathrm{Z}$, le lecteur par une digression symbolique la fait-il entrer en résonance avec un imaginaire de cavernes, bachelardien avant la lettre? Avec un imaginaire de conspiration févalien ? Avec une religiosité moins intertextuelle que symptômale? Un mot seulement sur ce possible chemin de traverse: la crypte-cathédrale souterraine des $\mathrm{Z}$ entrerait en résonance à la fois avec tout récit gnostique fondamental (malgré l'organisation sociale, malgré l'homme et malgré Dieu, il continue à y avoir le Mal) et avec la reconfiguration de la société historiquement déterminée induite par la promulgation le 9 décembre 1905 de la séparation des Églises et de l'État laïcisé. Reconfiguration qui aurait créé une ouverture pour un mouvement sectaire fictionnellement figuré par ces $\mathrm{Z}$, avec son idéologie dualiste, son objectif de parasitage criminel de la société, son ZigomarZarathoustra et son démiurge-trickster ${ }^{37}$...

49 Vadrouilleur laissant son esprit musarder à la moindre aspérité du texte lorsque ce dernier oublie de se faire oublier, de s'effacer pour mettre aussi directement que possible l'histoire sous les yeux du lecteur, ce dernier par une digression sociolinguistique entend-il les sorties du dialecte véhiculaire fasciculaire, depuis le jeu 
de mot bilingue («Broquet is broken !...», 10 janvier 1910) jusqu'aux variations dialectales (l'accent aveyronnais de Clafous, «Qu'est-che que je vas vous chervir ? [...] Y a-t-il longtemps là, qu'on ne ch'est pas vu!... Cha me fait plaijir !... », 5 janv. 1910), même si le récit fait tout pour aussitôt assujettir ces saillies faisant saillance, le jeu de mot narrativement justifié d'être un commentaire lapidaire, conclusif et prématuré proposé par le grand juge criminel à ses Ramogiz sur la scène qui vient de se dérouler, l'accent rouergat narrativement justifié d'être l'un de multiples artifices visant à dissimuler l'allégeance réelle de Clafous) ${ }^{38}$.

\section{Forte impression}

Dernier retour sur l'ambivalence de l'impression en régime médiatique, à la fois jetable et marquante, dernier retour sur l'oubli où Sazie et Zigomar ont été relégués et l'insistance mise ici par un lecteur à en rappeler l'attrait paradoxal... Zigomar a marqué, c'est pourquoi il a été imité par ses compétiteurs contemporains. Ainsi, comme Peau d'anguille paru en 1909-1910 et repris en volume en 1916 sous le titre Zigomar au service de l'Allemagne, L'Agent secret (1911), quatrième volume du «Fantômas » de Pierre Souvestre et Marcel Allain, articule l'univers de l'espionnage à un univers sériel préexistant. Une scène oppose les alliés Juve et Fandor: alors que le premier voit immédiatement dans le meurtre initial du capitaine Brocq la signature de Fantômas, le second, s'appuyant sur l'appartenance de la victime au Deuxième Bureau, voit tout aussi spontanément dans ce crime une affaire d'espionnage, chacun soupçonnant l'autre de s'adonner à sa marotte, aucun d'eux n'envisageant l'hypothèse (que pourtant la suite du récit va avérer) que les deux interprétations sont non pas contradictoires mais superposées. Toutefois l'arrimage entre les grands thèmes de la série et l'univers de l'espionnage s'avèrent faibles. L'air du temps et l'exemple de Sazie se chargeront de leur souffler une formule mieux intégrée: pourquoi ne pas construire une série entièrement consacrée à l'espionnage? Ce que Pierre Souvestre et Marcel Allain réalisent en 1912 et en quinze volumes, avec une histoire qui débute comme une classique variation sur un thème à la Jules Mary, la fragilité du militaire. Victime des machinations de l'espion Wolff et de son complice français Pajot, le lieutenant Frédéric de Mareuil est non seulement accusé d'espionnage par les Prussiens et de trahison par les Français mais aussi tenu responsable de l'assassinat de sa tante qui vivait en Lorraine occupée. Même si les malheurs continuent à lui pleuvoir sur la tête, l'officier reste plein d'initiatives; il a en effet reçu le soutien d'un ancien truand dont le destin n'est pas non plus dépourvu d'aléas, Naz-en-l'air. Même si les deux se partagent la vedette, c'est ce dernier qui donne son nom à la série, comme pour avouer par un discret $\mathrm{z}$ la dette à l'endroit du Zigomar espion, es/zpion. Sazie quant à lui avait abandonné ce terrain et n'y reviendra plus.

51 Or Zigomar a marqué parce qu'il a eu une importance déterminante dans la formation du roman d'espionnage. Mal prévisible en regard des romans qui l'avaient précédé, Zigomar au service de l'Allemagne mérite qu'on attire l'attention sur quelques unes de ses caractéristiques.

Revenons d'abord sur la structure en écheveau narratif, à la Ponson du Terrail, qui contribuerait à la fuite en avant diégétique peu soucieuse de cohérence, ce que confirmerait ce résumé du livre premier de Zigomar au service de l'Allemagne, «Pour la patrie et pour l'argent !", 
Le baron Otto Friedenstrasse, chef des espions allemands, annonce de but en blanc au marquis don Izigo Martolo qu'il se porte acquéreur du secret traité des Dardanelles dont Zigomar a le projet de s'emparer ; on s'entend pour la somme d'un million. Le baron-espion ne désespère toutefois pas de se procurer autrement le document pour une fraction de ce montant.

Au ministère, la belle comtesse Garitza obtient la combinaison du coffre où $M$. Desbois-Billy et son chef de cabinet, M. Simonet, enferment le brouillon du traité. Par ailleurs, un jeune attaché du ministère, Raymond est en train de tomber dans les rets du banquier James Benamol, alias Izigo Martolo, à cause de dettes de jeu. Ce dernier photographie nuitamment le document.

Schmidt, ancien légionnaire, au service de Friedenstrasse et de Zigomar, trahit l'un pour l'autre ; déguisé en Benamol, il est exécuté par ce dernier. Paulin Broquet, le fin limier, enquête ; le " vrai » Benamol lui semble suspect, mais lui-même est déjà filé. Le ministre se rend compte que son coffre a été violé. Broquet est aussi chargé de cette nouvelle enquête et, rapidement, établit un lien entre les deux affaires. Dans la rue, il reconnait le regard de son vieil ennemi, mais Zigomar disparaît, déguisé en camelot, filé par un policier.

Broquet perce peu à peu à jour la vraie personnalité de feu Schmidt ; dans son appartement, il trouve des mannequins, ceux de Benamol, d'une élégante entrevue au ministère, celui de l'innommable « Lui ». Dans la même maison, le capitaine Malther est espionné par Marguerite, la bonne allemande ; Broquet y est assommé.

Les capitaines La Rochardie et Montgomery consolent leur ami Simonet, lui assurant qu'ils ne croient à aucun manquement de sa part dans l'affaire du document photographié ; ils sont espionnés au restaurant. Ils y croisent don Izigo Martolo, la comtesse Pepa d'Orteza et La Roucoulière, un vieux beau qui fait le siège de l'élégante comtesse argentine, et sont invités chez elle. Le ministre veut lui aussi manifester à Simonet sa confiance : il le charge d'une mission secrète. Pendant ce temps, l'Amorce, autre collaborateur de Broquet, fait rapport sur la curieuse surveillance dont les trois jeunes gens sont l'objet, et sur le caractère tout à fait prémédité de la rencontre de la comtesse Pepa et de Simonet au restaurant. Gabriel et l'Amorce sont envoyés à la Taverne luxembourgeoise pour s'informer. Ils sont néanmoins vite repérés par Zigomar et le baron japonais Osaka-Itscho.

Broquet, ayant déguisé le chien de la malheureuse fiancée de Schmidt, déambule dans le quartier pour retrouver la piste de quelque éventuel collègue de l'espion assassiné et découvre que deux personnes assument alternativement la personnalité du vieux comptable Gutman, l'un détestant les chiens, l'autre les adorant. Au même moment, Mapipe, un autre adjoint de Broquet, de garde chez Schmidt, est endormi par un poison végétal émanant des pages d'un livre, laissant le champ libre à des hommes masqués qui vident l'armoire de l'espionphotographe de son contenu et laissent un funeste cadeau à Broquet : une bombe de cristal qui devrait immanquablement l'expédier ad patres. C'est, du moins, ce que Zigomar escomptait ; il croit Broquet mort, mais la bombe a heureusement 
atterri sur un lit ; il croit Mapipe mort aussi, mais Broquet lui a administré un contre-poison.

Fernande, sur son lit d'hôpital, trace avec son moignon sanglant le Z fatidique dénonçant Zigomar : elle venait de reconnaître la fausse infirmière qui allait l'achever. Insaisissable, le criminel s'enfuit de nouveau.

Jouant à affoler le cœur de Simonet, Pepa le convie de façon pressante chez elle, avec ses amis, avant son départ en mission à Londres. Dans un luxueux hôtel, un gentleman aux yeux de fauve, Will Wilson distribue blâmes et ordres à une assemblée hétéroclite de conjurés. Soirée chez la belle Pepa, où la jalousie du grotesque et amoureux La Roucoulière l'incite à provoquer Simonet en duel.

Broquet essaie de déjouer le piège que don Izigo et Pepa tendront sûrement au jeune homme dans son voyage vers Londres. Puis, il file Marguerite, la bonne allemande, pour repérer son contact - ses messages sont de fait acheminés par des œufs en celluloïd.

Simonet et ses amis sont attaqués dans le train par des $\mathrm{Z}$ en cagoule ; bagarre dans le compartiment, puis sur le toit des wagons où Broquet affronte Zigomar - tunnel, fumée noire, Zigomar disparaît, le train freine, poursuite, seuls deux bandits sont finalement capturés...

Outre les effets de confusion artefactuels générés par le résumé d'une portion seulement de l'ensemble de l'histoire (pour le lecteur de l'ensemble, nulle perplexité par exemple devant cette Fernande qui semble avoir atterri là comme un z sur un moignon), la multiplication des personnages et de leurs relations permet de narrer en passant d'un fil à l'autre en donnant l'impression d'un écheveau narratif, même si l'ensemble tient par une rection, elle-même à double détente selon le templum d'observation découpé : l'affrontement simple entre Broquet (et les siens) et Zigomar (dont les différents projets criminels aimantent différentes configurations de subordonnés et d'alliés) constitue la rection la plus générale ; deux documents secrets consécutifs excitent la convoitise des méchants, ce qui déclenche l'interprétant atteinte à la sûreté de l'État, constitue la rection propre de «Pour la patrie et pour l'argent ! » et de Zigomar au service de l'Allemagne, l'ensemble qui le subordonne.

Je voudrais suggérer qu'il ne s'agit plus simplement pour Sazie, de manière conservatrice, rétrograde, d'émuler la structure en écheveau narratif à la Ponson du Terrail mais de la mettre au service d'un autre univers, celui du spionspiel, de prendre acte de l'axiologie patriotique, simplette, manichéenne, pour aussitôt la qualifier, en faire sourdre les contradictions, en la décalant, en lui faisant ressentir l'attraction de l'univers propre du scélérat absolu. À leur intersection, narrativiser les effets produits par l'univers de Zigomar sur l'axiologie proposée par le discours revanchard introduit la complexité, la promeut en dimension obligée de la fiction d'espionnage. Prendre acte ? Friedenstrasse n'est que le dernier avatar de la malice sournoise prussienne, une nouvelle incarnation d'une menace dénoncée depuis la coûteuse Défaite de 1871 par un roman d'espionnage en voie de cristallisation, en phase avec une bonne fraction de la fiction populaire et du discours social. À ce fond, la nouvelle conjoncture politique issue de la signature de l'Entente cordiale entre France et Grande-Bretagne (1904) et du traité de paix de Portsmouth consacrant la victoire japonaise sur l'empire russe (1905) ajoute 
une première complexité : alliance britannique pour le camp de Broquet (plus encore que le message secret envoyé à Londres, c'est par la collaboration de Mac Binkerton avec Broquet et par l'acte de bravoure du capitaine La Rochardie qui lui gagne le cœur de Maud Montgomery que le roman manifeste cette alliance, dans le livre suivant, « Au plus fort!») et alliance japonaise pour celui de Friedenstrasse (petite complexité complémentaire puisqu'il s'avère que les amis de nos amis ne sont pas forcément nos amis).

55 Faire sourdre les contradictions? Telle serait la deuxième caractéristique notable de Zigomar au service de l'Allemagne. Dans sa diffraction fictionnelle de la politique internationale, le roman choisit d'en dynamiser inégalement les éléments. Dans cet univers de combattants de l'ombre aux alliances réversibles, à cette intersection des affaires d'États et du grand banditisme, à l'axiologie du discours revanchard s'en superpose une autre, ni contradictoire ni équivalente, décalée : un système qui promeut courage et loyauté en valeurs centrales, une axiologie plus militaire qu'idéologique, une relativisation de l'unanimisme ligne-bleue-des-Vosges. L'action se charge à la fois de bousculer les positions de départ et de rendre apparentes des failles initialement mal aperçues. Ainsi dans la scène où sont réunis Friedenstrasse, l'infirmier Schulbach, l'Australien Mc Relian, le banquier James Benamol, la comtesse Warsovia, Mme Garitza, des barons japonais à qui le gentleman aux yeux de fauve distribue blâmes et ordres : homogénéité lisse d'un regroupement fortement organisé de complices. Il suffit que survienne un spectacle extérieur, le défilé d'un régiment de marsouins pour qu'apparaisse une fêlure dans cette homogénéité apparente: là où le baron Friedenstrasse injurie, le baron Osaka-Itscho, qui avait combattu côte à côte avec les Français à Pékin, exprime son respect. En se développant, le récit poussera cette différenciation entre complices jusqu'à ses dernières conséquences. Les Japonais sont d'abord impressionnés par le courage de La Rochardie sous la torture. Puis, Broquet par celui de l'officier japonais qui, pour empêcher le contre-espionnage français d'accuser les camarades de son commando secret, sacrifie héroïquement une de ses mains alors que, tentant de s'emparer d'un nouveau détonateur, une innovation technologique déterminante, il est tombé victime d'un piège tendu par Broquet. Plus tard, OsakaItscho, sévère pour les piètres résultats conjugués de Friedenstrasse et de Will Wilson, sait à la fois reconnaître la valeur de ses adversaires, perd confiance en ses alliés et décide de les quitter. Enfin, Broquet sauve cet adversaire japonais du coup de poignard que Wilson-Zigomar lui destinait - coup qui dans la confusion aboutit dans le lard du baron prussien, ce qui déclenche la fuite sans gloire des conjurés. Morale de la fable : le camp de Broquet et du lecteur peut s'entendre avec de loyaux ennemis comme ces Japonais dont on partage les valeurs, le bushido.

56 En face, Zigomar est inflexible en matière de changement d'allégeance; il les châtie impitoyablement lorsque ce ne sont pas des alliés mais des subordonnés qui s'y risquent. On a vu le triste destin de Schmidt, l'ancien légionnaire; on a fait allusion à

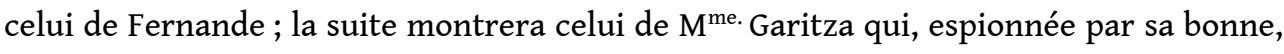
son double jeu découvert, subira la terrible vengeance de Zigomar. Voilà qui est simple et autorise la même ligne de conduite chez les adversaires : lorsque Broquet démasque Félan, son collaborateur félon, c'est intentionnellement et sans état d'âme qu'il l'envoie finir dans les flammes de la bombe à éther que Zigomar lui avait destinée. "C'était atroce, mais de bonne guerre... c'était aussi de toute justice » commente le roman (p. $290)^{39}$. 
Toutefois, cette simplicité n'épargne pas la contradiction à Broquet. D'un côté, au nom

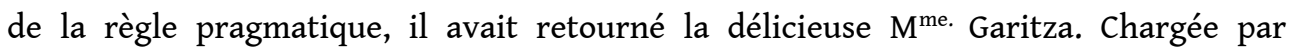
Zigomar de subtiliser les plans du détonateur à son soupirant militaire français, elle en est tombée amoureuse; Broquet, dissimulé, avait assisté à la scène dans laquelle le Maître, menaçant, reprochait à son employée de ne pas aboutir, avait facilement circonvenu l'amoureuse au profit du bon camp. En ce cas, la règle pragmatique semble s'accommoder de l'axiologie et la trahison devenir acceptable dans la mesure où elle se fait dans le bon sens (au profit des Français) et pour la bonne raison (l'amour). La trahison n'en sera pas moins compensée, par la mort de l'espionne retournée; et de manière particulièrement cruelle.

Ce qui conduit à la troisième caractéristique de Zigomar au service de l'Allemagne, dont le roman d'espionnage ultérieur, surtout après la Guerre froide, allait faire grand usage : l'effraction des corps ${ }^{40}$. Elle forme système avec le thème déjà mentionné, de l'élusive identité de Zigomar et des trompeuses apparences. Parmi bien d'autres, en voici deux exemples: Broquet, ayant déguisé le chien de la malheureuse fiancée de Schmidt, déambule dans le quartier pour retrouver la piste de quelque éventuel collègue de l'espion assassiné et découvre que deux personnes assument alternativement la personnalité du vieux comptable Gutman, l'un détestant les chiens, l'autre les adorant ; près de la conclusion de l'histoire, Broquet découvre qu'un $\mathrm{Z}$ alter-ego, tué d'un coup d'épée, jouait le rôle de don Izigo Martolo, ce qui fournissait à ce dernier un alibi lors des mauvais coups, lui qui était soupçonné, à juste titre, d'être aussi Zigomar (ce qu'un léger redécoupage de son nom aurait laissé deviner). Or, à cette époque, la progressive instauration de l'anthropométrie et de la photographie judiciaires d'Alphonse Bertillon comme méthodes d'identification criminelle étaient la réponse de la police à la nécessité de fixer l'identité des suspects et des criminels par le recours au corps représenté. Dernières années donc où la fiction peut rêver qu'il est possible pour les hors-la-loi de s'en tirer en se déguisant et en se grimant. Par la représentation, le corps du bertillonage remplace le marquage direct; l'essorillage, le marquage au fer rouge, la brûlure de la bouche ne sont plus permis. Chez Sazie, le corps ne se réduit pas à cette problématique d'identité ; c'est à lui que s'ente la cruauté.

Cruauté assez riche et diversifiée pour se voir pourvue de plusieurs fonctions. Mains tranchées de Fernande, de l'officier japonais, espion allemand ébouillanté dans la baignoire par La Rochardie... : l'altération du corps marque, la lecture allegro appelée par le toboggan diégétique en est désunie, comme on dit du trot d'un cheval. Mais le récit peut aussi l'assujettir à l'univers du spionspiel, l'instrumentaliser. Ainsi cette effraction du corps concluant la soirée à l'Opéra où sont rassemblés tous les protagonistes, soirée qui se termine dramatiquement puisque Simonet est poignardé et que Broquet retrouve sur lui le message secret du Foreign Office. Trahissait-il ? Broquet flairant un montage de Zigomar va au chevet du blessé avec le ministre Desbois-Billy, renifle le parfum blond de la comtesse Warsovia, se saisit d'Adolphe, le valet de chambre de Simonet (mais aussi bien vicomte et ami du marquis don Izigo!) : c'était bien la comtesse qui avait glissé les documents incriminants dans l'habit de Simonet, à son insu. La tentative de meurtre au service d'une entreprise d'intoxication. L'effraction du corps peut encore aspirer la lecture comme un maelström - ainsi lorsque Zigomar en Bossuet sadique annonce à sa victime les effets à venir du poison qu'il vient de lui administrer :

Attendez !... Vous êtes jeune... vous êtes jolie...vous êtes blonde exquise... Votre peau est comme du satin. Votre corps est une merveille de forme... Vous êtes 
adorable... on vous adore !... Tous ceux qui ont eu le bonheur de vous aimer ont gardé de vous un souvenir enchanteur et que nulle autre femme n'a pu effacer...

- Quel poison? redemanda Mme Garitza

- Eh bien, vos cheveux vont tomber, votre peau va se marbrer, s'écailler, vos membres vont se déformer... et partout vont, de votre corps, surgir des boutons en grappes qui gonfleront comme des raisins au soleil... se fendront... laisseront couler un pus verdâtre... Vos doigts artistes, musiciens, caresseurs tomberont un à un et vos pieds se détacheront par morceaux... Vos mains partiront... votre corps entier s'en ira en lambeaux... Vous serez tellement horrible... tellement épouvantable, que même le colonel qui vous adore - à moins d'être plus grand qu'un héros humain n'aura pas le courage de s'approcher de vous... Il ne vous restera dans cette ruine, que vos yeux, si bleus, pour voir la chose épouvantable et sans nom que la belle madame Garitza sera devenue !... (p. 305-306) du temps, le bourreau ironise «Ces Français, tous les mêmes! Quand on presse un peu dessus, ils s'aplatissent!» (p. 190). Sans ironie, le lecteur comprend, lui, tremblant, la forte impression exercée par cette presse, face ésotérique de la matérialité du travail médiatique d'impression, instrument qui permet, comme Zigomar ou comme l'illustration de couverture, d'annoncer au chaland à quoi s'en tenir, de saisir l'action à son acmé. Létale pression sur le corps d'un personnage qui marque le tourniquet de l'impression pour le lecteur : la presse, celle où paraît son roman-feuilleton ou celle qui en a imprimé le livre, a permis d'imprimer cette intranquillité à son esprit frissonnant mais aussi réussi à confiner la méchanceté en une fiction, dans le livre refermé.

BIBLIOGRAPHIE 


\section{Études}

Avenel, Henri. La Presse française au vingtième siècle, Paris, Flammarion, 1901.

Baldick, Robert.The Duel: a History of Duelling, London, Chapman and Hall, 1965

Barthes, Roland. S/Z, Paris, Seuil, « Tel Quel », 1970.

Becker-Ho, Alice. Les Princes du jargon : un facteur négligé aux origines de l'argot des classes

dangereuses, Paris, Gallimard, 1993.

Becker-Ho, Alice. L'Essence du jargon, Paris, Gallimard, 1994.

Bianchi, Ugo. « Le dualisme en histoire des religions », Revue de l'histoire des religions, vol. 159, $\mathrm{n}^{\circ} 1$, p. 1-46, 1961 - disponible à http://www.persee.fr/web/revues/home/prescript/article/

rhr_00351423_1961_num_159_1_7599

Bleton, Paul. Ça se lit comme un roman policier... Comprendre la lecture sérielle, Québec, Éditions Nota bene, « Études culturelles », 1999.

Bleton, Paul. Les Anges de Machiavel. Essai sur l'espionnage, Québec, Nuit blanche éditeur, « Études paralittéraires ", 1994.

Bourget, Jean-Loup. Le Mélodrame hollywoodien, Paris, Stock, « Cinéma », 1985.

Chevalier, Louis. Montmartre du plaisir et du crime, Paris, R. Laffont, « Les Hommes et l'histoire », 1980.

Couégnas, Daniel. « Les charmes du récit interminable : Rocambole, de Ponson du Terrail », Fictions, énigmes, images, Limoges, PULIM, « Médiatextes », 2001.

Dixmier, Michel et Véronique Willemin, L'Eil de la police. Crimes et châtiments à la Belle Epoque, Paris, éditions Alternatives, 2007.

Jeanneney, Jean-Noël. Le Duel, une passion française, 1789-1914, Paris, Éd. du Seuil, 2004.

Kalifa, Dominique. « Zigomar, grand roman sériel (1909-1913) » dans Crime et culture au XIX ${ }^{e}$ siècle, Paris, Perrin, « Pour l'Histoire », 2005.

Landowski, Benjamin. Le Duel devant la loi, Paris, impr. de J. Dangon, 1904.

Lenoble, Benoît. « Les campagnes de lancement de romans-feuilletons : l'exemple du Journal (1892-1935) », Revue d'Histoire moderne et contemporaine, n52, janvier-mars 2005.

Le Ray, Éric. Marinoni. Le Fondateur de la presse moderne (1823-1904), Paris, L'Harmattan, « Graveurs de mémoire », 2009.

Letainturier-Fradin, Gabriel-Jules-Adrien-Paul (pseud. Gabriel d'Arcy). Le Duel à travers les âges, histoire et législation, duels célèbres, code du duel, préf. de A. Tavernier, Paris, E. Flammarion, 1892.

Mellot, Philippe. Les Maîtres du mystère, de Nick Carter à Sherlock Holmes, 1907-1914, Mantes-la-Jolie, éditions Michèle Trinckvel, 1997.

Muchembled, Robert. Une Histoire de la violence, Paris, Seuil, « L'univers historique », 2008.

Neveu, Erik. L'Idéologie dans le roman d'espionnage, Paris, Presses de la Fondation nationale des sciences politiques, 1985.

Singer, Ben. Melodrama and Modernity. Early Sensational Cinema and its Contexts, NY, Columbia UP, "Film and Culture", 2001. 
Stedman, Raymond W. The Serials. Suspense and Drama, Norman, University of Oklahoma Press, 1977.

\section{Pièces}

Gandillot, Léon. Les Femmes collantes, comédie-bouffe en 5 actes [Déjazet, 16 octobre 1886], Paris, P. Ollendorff, 1886.

Gandillot, Léon. La Course aux jupons, comédie en 3 actes [Déjazet, 20 février 1890], Paris, P. Ollendorff, 1890.

Gandillot, Léon. Bonheur à 4, comédie en 3 actes[Vaudeville, 25 mars 1891], Paris, P. Ollendorff, 1891.

Gandillot, Léon. La Mariée récalcitrante, comédie-bouffe en 3 actes [Déjazet, 19 janvier 1889], Paris, P. Ollendorff, 1893

Gandillot, Léon. Associés ! comédie en 3 actes [Déjazet, 26 novembre 1894], Paris, P. Ollendorff, 1895.

Gandillot, Léon. Ferdinand le noceur, comédie en 4 actes [Palais-Royal, 1896].

Sazie, Léon. Bisbis de ménage, comédie en 1 acte, Paris, Librairie théâtrale, 1893.

Sazie, Léon. Un Gendre au cassoulet, comédie en 1 acte, Paris, Librairie théâtrale, 1893. Sazie, Léon. La Sonate en mi, comédie en 1 acte [Gymnase, 2 octobre 1892], Paris, Librairie théâtrale, 1893.

Sazie, Léon. " Je suis discrète », dans Le Livre des petites filles, Paris, Librairie théâtrale, 1893.

Sazie, Léon et Georges Grison. Jacques l'honneur, drame en 5 actes et 9 tableaux [République Château-d'Eau, 30 octobre 1894], Paris, Librairie théâtrale, 1895.

Sazie, Léon et Georges Grison. Caponet, Pipard et Cie, vaudeville en 3 actes [création à Bruxelles 29 sept. 1896, repris à Paris au théâtre de la Bodinière, 1899]

Sazie, Léon. Larmes d'or et gâteau de plomb, récit pour jeunes filles, Paris, Librairie théâtrale, 1898.

\section{Films}

Bertoni, Albert-Francis, Enfants de Paris, d'après [Francis Didelot et] Léon Sazie, avec Félicien Tramel et Marguerite Madys, 1924

Bourgeois, Gérard. Les Mystères du château de Malmort, avec Josette Andriot, Éclair, 1917.

Caron, Pierre. Les Femmes collantes, d'après Léon Gandillot, , avec Henri Garat, 1938.

Faivre, Jean. Protéa et l'auto infernale, avec Josette Andriot, Éclair, 1914.

Faivre, Jean. La Course à la mort, avec Josette Andriot, Camille Bardou, Éclair, 1915.

Jasset, Victorin. Protéa, avec Josette Andriot, Lucien Bataille, Éclair, 1913.

Monca, Georges. La Mariée récalcitrante, d'après Léon Gandillot, 1916

Monca, Georges. Ferdinand le noceur, d'après Léon Gandillot, 1913.

Monca, Georges. Les Femmes collantes, d'après Léon Gandillot, 1920

Renaud, Jean-Joseph. L'Intervention de Protéa, avec Josette Andriot, Henri Maillard, Éclair, 1918. 
Roudès, Gaston, Enfants de Paris, d'après Francis Didelot et Léon Sazie, sc. de Didelot, avec Paul Bernard, Lisette Lanvin, 1936.

Sti, René. Ferdinand le noceur, d'après Léon Gandillot, avec Fernandel, 1935.

Romans, nouvelles, contes

Anon. Ferdinand le noceur, Paris, Le film complet, $\mathrm{n}^{\circ} 1670,1935$.

Anon. Les Femmes collantes, Paris, Le film complet, $\mathrm{n}^{\circ} 2139$, Paris, 1938.

Allais, Alphonse. Le Parapluie de l'escouade, Paris, Ollendorff, 1893.

Allais, Alphonse. Rose et vert pomme, Paris, Ollendorff, 1894.

Galopin, Arnould. Ténébras, le bandit fantôme, Paris, Librairie Contemporaine, 1911.

Gandillot, Léon. Contes à la lune, Paris, Librairie illustrée, [1888].

Gandillot, Léon. Les Femmes collantes, roman drôlatique inédit, illustrations Gil Baer, Paris, J. Rouff, (s. d.)

Leblanc, Maurice. L'Aiguille creuse, Paris, P. Lafitte, 1909.

Leblanc, Maurice. 813, $1^{\mathrm{e}}$ partie, La Double vie d'Arsène Lupin, Paris, P. Lafitte, 1911.

Leblanc, Maurice. 813, 2e partie, Les Trois crimes d'Arsène Lupin, Paris, P. Lafitte, 1917.

Saize, Noël de. La Vénus des palaces, Paris, Arthème Fayard, « Les Maîtres du roman populaire », $n^{\circ} 461,1933$.

Saize, Noël de. Tonico, danseur mondain, Paris, Arthème Fayard, « Les Maîtres du roman populaire ", $n^{\circ} 478,1934$.

Sazie, Léon et Georges Grison. Jacques l'Honneur, roman dramatique, Paris, H. Geffroy, « Romans illustrés », 1895.

Sazie, Léon. Enfants de Paris, roman d'amour abondamment illustré par les photographies du film Grandes Productions Cinématographiques, Paris, Tallandier, « Cinéma bibliothèque », nº 93, 1924.

Sazie, Léon. Un Nouveau coup de Zigomar, Paris, Tallandier, « Le Livre national rouge », n 16, 1948.

\section{NOTES}

1. Je cite la réédition dans la collection Points, $n^{\circ} 70,1976$.

2. Trois bobines d'une dizaine de minutes chacune.

3. Arquillère [Paul Perret] avait débuté en 1888, acquis une longue expérience de la scène en participant notamment à l'expérience du Théâtre libre Antoine ; c'est à partir de 1908 qu'il a commencé à tourner.

4. Au cinéma, Liabel devait surtout réaliser, notamment l'adaptation d'un roman de Valentin Mandelstamm, Le Secret d'Alta Roca (1920) et de La Closerie des genêts (1924) d'après un célèbre mélodrame de Frédéric Soulié.

5. Actrice-fétiche de Jasset, elle devait incarner Protéa, l'espionne au collant (future inspiration pour l'Irma Vep de Feuillade) dont seul le premier a été réalisé par Jasset, les quatre suivants datant d'après le décès du réalisateur original (de 1914 à 1918).

6. Et non du roman homonyme de William Cobb, alias Jules Lermina, paru en 1874.

7. Sur l'entente de Pathé avec la presse Hearst, cf. Raymond W. Stedman (1977) ou Ben Singer (2001). 
8. Variation sur la définition que Jean-Loup Bourget (1985) propose à partir de la forme classique du mélodrame hollywoodien, plus tardive (1930-1960).

9. Après la disparition de Jasset et le succès de son Protéa (1913), Éclair refait quatre fois appel à la contre-espionne : avec Jean Faivre comme réalisateur pour Protéa et l'auto infernale (1914) et La Course à la mort (1915), à Gérard Bourgeois pour Les Mystères du château de Malmort (1917) et à JeanJoseph Renaud pour L'Intervention de Protéa (1918). Nouvelle malchance, posthume, pour cet héroïne de Jasset, supplantée à son tour par l'Irma Vep des Vampires de Feuillade.

10. Photo que l'on peut consulter sur http://www.paris.fr/loisirs/Portal.lut? pagemultimedialist\&page_id=102\&id=6

11. Échec peu représentatif de Gandillot, vaudevilliste à succès (notamment avec La Course aux jupons, 1890 ; Bonheur à 4, 1891 ; Associés! 1894), joyeux bohème, ex-centralien passé à l'école du Chat noir de Salis (cabaret dont il fut l'archiviste), fin conteur (si l'on en juge par ses contes et nouvelles disséminés dans l'Illustration, la Vie populaire, les Annales politiques et littéraires, ou réunis dans ses Contes à la lune, 1888), lui-même personnage de nouvelles et anecdotes d'Alphonse Allais (dans Le Parapluie de l'escouade, Ollendorff, 1893, Rose et vert pomme, Ollendorff, 1894). Tôt disparu (1862-1912), certaines de ses comédies burlesques devaient largement lui survivre grâce au cinéma. La Mariée récalcitrante (1889) sera portée à l'écran par Georges Monca en 1916 ; Ferdinand le noceur (1896) par Monca en 1913 puis par René Sti en 1935, avec Fernandel, film lui-même aussitôt novellisé en ciné-roman; Les Femmes collantes (1886), déjà gonflé en un long "roman drôlatique » illustré (par Gil Baer) en 2 tomes de plus de 2000 in- $8^{\circ}$ chacun chez Jules, par Monca en 1920 puis Pierre Caron en 1938, avec Henri Garat, film lui aussi aussitôt novellisé en cinéroman.

12. Des adaptations en BD quotidienne dans France-Soir, illustrée par Henry Blanc: 1174 strips (1957-1960) - eux-mêmes réédités en deux volumes par Pressibus (1999) -, en romans chez J.-C. Lattès (Malheur aux Barbus, Le Boudin sacré, Crimée...châtiment, La Lumière qui éteint, L'Atoll Anatole, M...comme..., 1981), à l'écran par Marc Simenon (la deuxième saison) Signé Furax : le film (1981). Il devait aussi y avoir rediffusions radiophoniques (en 1968 et en 1988), suites sous forme de disques 45 tours - Menace sur Tancarville, La Navrante comédie des proverbes, Trafic de larmes, L'Enlèvement du Père Noël, Alerte aux homards (1960) elles-mêmes rééditées sous forme de cédéroms (2005) - et exploitation sous forme de cassettes puis de cédéroms (2005).

13. Fantômas (1964), Fantômas se déchaîne (1965) et Fantômas contre Scotland Yard (1967).

14. Prolifique illustrateur souvent apparié à Galopin, notamment, et dans un style graphique très différent, pour la série SF des Aventures fantastiques d'un jeune parisien (1908). L'éditeur, la Librairie Contemporaine, recueillera le tout en un volume sous une couverture illustrée par Robert Sallès d'un homme, un mondain aux yeux fous, pointant son revolver sur le lecteur.

15. Suite de L'Algérie juive (1887).

16. Environ 227700 euros.

17. Le décret $n^{\circ} 409$ précise « situées dans le territoire des Ouled-Maziz, district de Lalla-Maghnia (département d'Oran)», p. 560.

18. Disciple d'Auguste Comte, notamment sur l'idée d'unité de la science, pédagogue - répétiteur de mécanique à l'École Polytechnique, qui en plus de ses ouvrages mathématiques, a aussi fait paraitre La Mathématique. Philosophie. Enseignement (1898) - et fondateur de la revue savante l'Intermédiaire des mathématiciens.

19. Brindeau a co-fondé l'Association des peintres et sculpteurs du Maroc, fondé un musée des Arts populaires à Casablanca, exposé aux expositions coloniales en France...

20. Sur la place symbolique de l'escrime et du duel, on peut lire aussi bien des textes contemporains de Sazie - de l'essai juridique à la synthèse historique, comme Le Duel devant la loi (1904) de Benjamin Landowski ou Le Duel à travers les âges, histoire et législation, duels célèbres, code du duel (1892) de Gabriel-Jules-Adrien-Paul Letainturier-Fradin - que des études plus proches de 
nous et plus loin de cette "passion française» comme Robert Baldick (1965) ou Jean-Noël Jeanneney (à qui est justement empruntée cette expression).

21. Journaliste au Figaro, qui avait couvert notamment l'exposition universelle de 1889 ; il devait publier quelques romans populaires.

22. Pour s'en faire une idée, cf. Michel Dixmier et Véronique Willemin (2007).

23. Cf. Benoît Lenoble (2005).

24. La Vénus des palaces (1933) et Tonico, danseur mondain (1934).

25. Sur l'acte de lecture, cf. Paul Bleton (1999).

26. Pour une idée générale des campagnes de lancement des romans-feuilletons, cf. Benoît Lenoble (2005). Il prend l'exemple du Journal (1892-1935).

27. Comme Le Yacht blanc et les huit fascicules de La blonde aimée.

28. Ainsi que le suggère Matthieu Letourneux.

29. La parution avait commencé avec L'Héritage mystérieux dans la Patrie en 1857 et s'était achevée avec La Corde du pendu dans la Petite Presse en 1870 ; mort prématurément en 1871 Ponson laisse le cycle inachevé. Dentu en avait assuré la publication en volumes (1866-1870), puis Rouff (1884); avec les 46 volumes des Cuvres de Ponson du Terrail, Arthème Fayard ne prenait pas un gros risque éditorial.

30. Pour ces caractéristiques, je reconnais ma dette à l'endroit de l'analyse de l'esthétique de Rocambole proposée par Daniel Couégnas (2001) .

31. Je cite ici le roman-feuilleton du Journal, numérisé sur Gallica.

32. Allusion à Alice Becker-Ho (1993 et 1994).

33. Sur Marinoni, cf. Éric Le Ray (2009).

34. Il allait s'en inspirer pour créer Arsène Lupin en 1905.

35. Le feuilleton hésite entre cette orthographe et une autre, Guairinière.

36. Pour ce dernier cas, cf. cette scène de vaudeville où l'auteur exhibe sa mauvaise foi, confessant explicitement à son lecteur qu'il ne dit pas tout ce qu'il sait: "Le rat d'hôtel continuons à le désigner ainsi pour plus de clarté dans le récit, afin de nous reconnaître dans ce jeu de portes [...]» (26 déc. 1909). Sans enjeu pour l'action, l'énigme sur son identité et celle des autres visiteurs nocturnes clandestins de cet hôtel est rapidement résolue : l'affaire impliquait, le lecteur apprend-il bientôt, Broquet et ses deux assistants, l'Amorce et Gabriel...

37. L'hypothèse de la parenté du démiurge gnostique avec le trickster des ethnologues, dans des aires où le créateur éprouve une insuffisance au moment de la fondation ou dans son gouvernement, est explorée par Ugo Bianchi (1961).

38. L'intérêt de Sazie, né en Algérie, pour la variation sociolinguistique dans la langue maternelle s'était tôt manifesté dans une nouvelle, «Bou-Zian et la poupée », où il faisait parler un turco algérien - on retrouve en Sidi, un adjoint algérien de Broquet, un écho de cette nouvelle. Henri-Yvon Mermet et Claude Mesplède parlent d'études à Orthez; on retrouve aussi un Léon Sazie à l'école militaire de Sorèze dans le Tarn entre 1877 et $1880 .$.

39. Je cite maintenant Zigomar au service de l'Allemagne (1916).

40. Cf. Erik Neveu (1985), Paul Bleton (1994). 


\section{RÉSUMÉS}

Zigomar ? Oui, car c'est le succès médiatique de ce mystérieux criminel oublié (seul son nom a survécu) qui avait servi de modèle à Souvestre et Allain. Son auteur, Léon Sazie, est encore plus oublié ; il était temps de faire un bref rappel de ce que l'on sait de ce romancier mis à l'ombre par son féroce et insaisissable héros. Mais surtout, en suivant le fil rouge et entrelacé de l'impression, il était temps de resituer l'invention dans son contexte et d'en restituer l'impact sur ses lecteurs d'alors.

Zigomar? Yes, because the media success of this forgotten mysterious criminal (nowadays only his name is remembered) has made him a model for Souvestre and Allain. Léon Sazie is even more forgotten. It is high time to sum up what we know about him. And high time to replace that type (the absolute criminal) in its historical context and remind the commotion Zigomar had on his readership.

\section{INDEX}

Mots-clés : Sazie Léon, Zigomar, transmédialité, intermédialité, récit criminel, culture médiatique, Fantômas

\section{AUTEUR}

\section{PAUL BLETON}

Paul Bleton est Professeur à la TéLUQ (Télé-université, Montréal) depuis 1982, il y a conçu des manuels sur la communication écrite et l'argumentation, la linguistique et la philosophie du langage, la communication non-verbale et la communication interculturelle, la littérature de grande diffusion et la bande dessinée. Il a écrit sur le roman d'espionnage Les Anges de Machiavel (1994) et La Cristallisation de l'ombre (2010), sur l'imaginaire français de la conquête de l'Ouest, Western, France (2002). Une autre étude, Combats. Récit de guerre et imaginaire militaire en France, est à paraître. Il a aussi publié un essai sur l'acte de lecture propre au roman populaire, Ça se lit comme un roman policier... (1999). Il a co-signé Lignes de front. Le Roman de guerre africain (2009) avec Désiré Nyela et Le Vagabond stoïque, Louis Hémon (2004) avec Mario Poirier. Il a été le maître d'œuvre de plusieurs numéros thématiques de revues (comme Belphégor, Études littéraires, Protée, Les Cahiers pour la littérature populaire...), dirigé plusieurs volumes collectifs : Hostilités. Guerre, mémoire, fiction et culture médiatique (2001), Amour, aventure et mystère ou : les romans qu'on ne peut pas lâcher (1998), Les hauts et les bas de l'imaginaire western (avec Richard Saint-Germain, 1997), Armes, larmes, charmes. Sérialité et paralittérature (1995) et publié de nombreux articles sur la fiction populaire. 